\title{
I2V Highway and Urban Vehicular Networks: A Comparative Analysis of the Impact of Mobility on Broadcast Data Dissemination
}

\author{
Stefano Busanelli, Gianluigi Ferrari, and Vito Andrea Giorgio \\ Wireless Ad-hoc and Sensor Networks (WASN) Lab, Dept. of Information Eng., University of Parma, Italy
}

\begin{abstract}
In this paper, we consider the problem of disseminating data in Infrastructure-to-Vehicular (I2V) IEEE 802.11 networks. We analyze, with a comparative approach, the performance in highway and urban scenarios. In particular, after characterizing the mobility in these scenarios we analyze the performance in terms of data dissemination from a fixed Road Side Unit (RSU) to the vehicles passing in its proximity through a recently proposed multihop probabilistic broadcasting protocol, namely Irresponsible Forwarding (IF). In the case of highway-like Vehicular Ad-Hoc NETworks (VANETs), we first characterize a mobile scenario in such a way to make a direct comparison with a static scenario meaningful, taking into account a physical characterization of the network (e.g., in terms of vehicle spatial density). Then, we consider a few mobile urban scenarios, characterized by the presence of junctions regulated by Traffic Lights (TLs) and Roundabouts (Rs). Our results show that, from a single packet perspective, the vehicles' mobility does not affect the behavior of the IF protocol, at least in the considered mobile scenarios (both highway and urban). However, different conclusions are reached when an information flow (i.e., a series of consecutive packets) is considered. In this context, we determine the maximum amount of data which can be transferred from the RSU to the mobile vehicles passing through a certain Region Of Interest (ROI) around the RSU.
\end{abstract}

Index Terms-Vehicular Ad hoc NETwork (VANET), Infrastructure-To-Vehicle (I2V), data dissemination, highway VANET, urban VANET, VANET simulators.

\section{INTRODUCTION}

Vehicular Ad-hoc NETworks (VANETs) are multipurpose networks suitable for a plethora of applications (e.g., safety, data dissemination, data collection, infotainment) [1]. In order to meet the needs of all these applications, the most relevant international standards, namely the WAVE-IEEE 802.11p [2] and the ISO Communications, Air-interface, Long and Medium range (CALM) [3], rely on both unicast and broadcast (often geocast) protocols. Unicast and broadcast strategies are complementary. For instance, while a multi-hop broadcast protocol fits well with applications like the diffusion of public interest

This paper is partially based on "On the Effects of Mobility for Efficient Broadcast Data Dissemination in I2V Networks," by S. Busanelli, G. Ferrari, and V. A. Giorgio, which appeared in the Proceedings of the IEEE Globecom 2010 SWiM Workshop, Miami, FL, USA, December 2010. (C) 2010 IEEE

The work of Vito Andrea Giorgio is supported by the Spinner consortium.

Manuscript received August 15, 2010; revised November 15, 2010; accepted January 15, 2011 messages (e.g., warning messages), the requirements of other applications (e.g., parking reservation) could be better satisfied by a unicast multi-hop protocol. Therefore, a general VANET usually needs to rely on both families of protocols.

With respect to other types of Mobile Ad-hoc NETworks (MANETs), one of the distinguishing features of VANETs is given by the peculiar mobility behavior of the nodes that constitute the network, influenced by both the road infrastructure and by the physical and socio-psychological factors that govern vehicles' interactions. In the last decades, mainly in the realm of road engineering, several models able to capture the vehicles mobility behavior have been proposed, such as car-following, stochastic, traffic stream, and flowsinteraction [4]. Among them, car-following models are probably the most common and the most realistic [5]. They were first employed in the 50's and are continuousin-time microscopic models, able to determine the behavior of each vehicle on the basis of the states (in terms of position, speed, acceleration) of the surrounding vehicles. In the field of VANETs, the performance of (peer-topeer) routing protocols has been evaluated according to two main indicators: the vehicles' (relative) speeds and the vehicle spatial density [6]. While these indicators are relevant, in the context of routing, to determine the lifetime of a multihop communication path, they become relevant, in the context of broadcasting, to determine the maximum transferable amount of data between a fixed source (Road Side Unit, RSU) and the vehicles positioned in a given Region Of Interest (ROI).

In this work, we focus on data dissemination in VANET with a probabilistic multihop broadcast protocol, named Irresponsible Forwarding (IF), originally proposed in [7] and applied, in [8], in IEEE 802.11 networks. Our goal is to investigate how the mobility affects data dissemination, in realistic (highway and urban) VANET scenarios. In order to carry out this investigation, here we compare, through simulations, the performance of IF in many radically different mobility conditions: a static highway VANET (e.g., a scenario where all cars move at the same speed); a mobile highway VANET in stationary conditions; several mobile urban VANET scenarios, with junctions controlled by Traffic Lights (TLS) and Roundabouts (Rs). The performance analysis is carried out, first, from a single packet prospective (meaningful for safety- 
related applications) and, then from an information flow prospective. In this latter case, we evaluate the maximum amount of transferable data, in terms of throughput, from the RSU to the vehicles lying in a ROI around a fixed RSU. As expected highway scenarios allow the transfer of a larger amount of data. In urban scenarios, the presence of junctions with Rs seems to be more limiting than the presence of TLs.

The structure of this paper is the following. In Section II, we introduce the VANET scenarios considered in our simulation analysis. Section III is dedicated to the characterization of the used mobility models. In Section IV, we describe the IF protocol, the considered performance metrics, and the simulation setup. In Section V and Section VI, we evaluate the per-packet and the per-flow system performance, respectively. Finally, conclusions are drawn in Section VII.

\section{REFERENCE SCENARIOS}

In [9], the performance of IF in a multiple-lane bidirectional highway was considered. We now extend this analysis to encompass also several mobile urban scenarios constituted by a few consecutive road intersections and, therefore, characterized by a non-homogeneous vehicle distribution. The highway scenarios are described in Subsection II-A (mobile) and Subsection II-B (static), whereas the urban scenarios are described in Subsection II-C. Despite their differences, the considered scenarios have the following common features.

- Each vehicle is equipped with an omni-directional antenna and is characterized by a fixed transmission range, denoted as $z$ (dimension: [m]).

- Each vehicle is equipped with a Global Positioning System (GPS) receiver. Therefore, we assume that each vehicle knows its own position at any given time.

- Each vehicle has the same length $L_{\mathrm{v}}=5 \mathrm{~m}$.

\section{A. Mobile Highway Scenario}

The reference mobile highway scenario is shown in Figure 1 and will be denoted, in the following, as $H_{\text {mob }}$. The road is composed by $N_{\text {lane }}=6$ adjacent lanes $(3$ per direction of movement), each with width equal to $w_{\text {lane }}=$ $4 \mathrm{~m}$. As indicated in Figure 1, we consider a portion of a highway whose length $L$ is set proportionally to the nodes' transmission range as follows: $L \triangleq \ell_{\text {norm }} z$, where $\ell_{\text {norm }}$ is an adimensional factor. The vehicles in the three lanes at the bottom are directed towards right (eastbound), whereas the vehicles in the upper three lanes are directed towards left (westbound).

The ROI is defined as the region centered around the RSU and with length $L_{\mathrm{ROI}}=\ell_{\mathrm{ROI}} z$, where $\ell_{\mathrm{ROI}}<$ $\ell_{\text {norm}}$. All the nodes lying in the ROI are implicitly interested in the reception of the packets generated by the RSU. We assume that the highway operates under stationary and stability conditions. In other words, the entering flux of vehicles in the ROI is the same of the

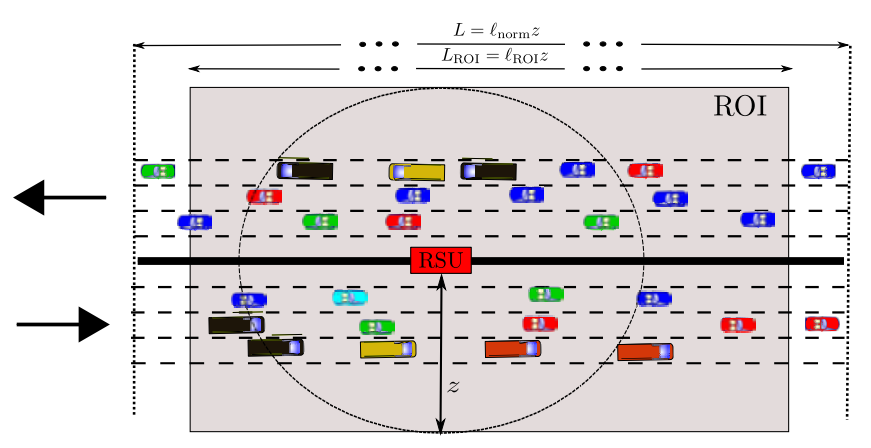

Figure 1. The mobile linear network topology in an highway scenario.

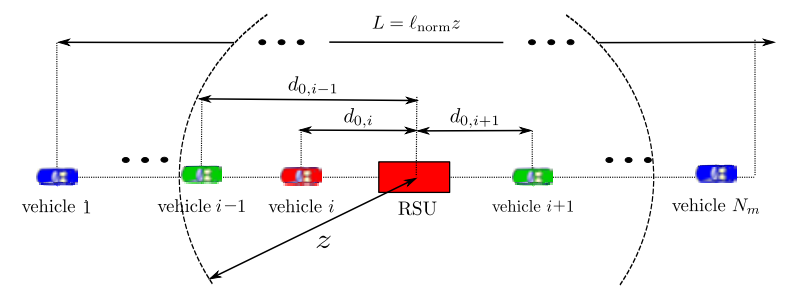

Figure 2. The linear network topology in a single lane of a highway scenario.

exiting flux of vehicles: more precisely, when a node exits from the network area, it is assumed to re-enter instantaneously. The number of vehicles in the ROI is the random process $N_{\mathrm{ROI}}(t)$.

Under the above assumptions, it is possible to define the instantaneous linear vehicle spatial density within the ROI as $\rho_{\mathrm{s}}^{\mathrm{R}}(t) \triangleq N_{\mathrm{ROI}}(t) / L_{\mathrm{ROI}}$ (dimension: [veh/m]). Conversely, the time-independent linear vehicle spatial density (dimension: $[\mathrm{veh} / \mathrm{m}]$ ) within the whole highway section is

$$
\rho_{\mathrm{s}} \triangleq \frac{N}{L}
$$

We stress the fact that the highway portion depicted in Figure 1 will be considered as a quasi-monodimensional network, ${ }^{1}$ since the width of the road will be much shorter than the transmission range $\left(z \gg N_{\text {lane }} \cdot w_{\text {lane }}\right)$.

\section{B. Static Highway Scenario}

A static highway scenario can be obtained by taking a snapshot of the mobile scenario or, equivalently, using the single-lane static model introduced in [8]. An $N_{\text {lane }}$ static scenario could be obtained by simply replicating $N_{\text {lane }}$ times a single lane. This scenario is denoted as $\mathrm{H}_{\text {sta. }}$. A representative realization of the topology of any lane of the highway is shown in Figure 2. In this case, since border effects can be neglected, the length of the highway section is $L=\ell_{\text {norm }} z=\ell_{\mathrm{ROI}} z$. There is a single source RSU, placed in the center of the network and identified by the index 0 . The $m$-th lane ( $m \in\left\{1,2, \ldots, N_{\text {lane }}\right\}$ ) contains $N_{m}$ nodes (each node is uniquely identified by an index $j \in\left\{1,2, \ldots, N_{m}\right\}$ ), where $N_{m}$ is a random variable. The positions of the $N_{\mathrm{m}}$ nodes are determined by a monodimensional Poisson point process with parameter

\footnotetext{
${ }^{1}$ In Figure 1, for illustration purposes the scale is not realistic.
} 
$\rho_{s} / N_{\text {lane }}$ (dimension: [veh/m]), where $\rho_{\mathrm{s}}$ is the vehicle linear spatial density defined in Subsection II-A. The validity of this assumption is confirmed by empirical traffic data [10]. By replicating this process $N_{\text {lane }}$ times, we deploy in the highway a random number of vehicles, given by the superposition of $N_{\text {lane }}$ Poisson point processes. By the property of the superposition of Poisson processes, the overall random process (in the horizontal axis) is still a Poisson process of parameter $\rho_{s}$. For comparison purposes, we assume that overall number of vehicles in the $N_{\text {lane }}$ lanes is given by the random variable $N$, as in the mobile case. In other words, we assume that

$$
N=\sum_{n=1}^{N_{\text {lane }}} N_{m} .
$$

\section{Mobile Urban Scenarios}

While a highway can easily be represented by a multilane straight road, a meaningful representation of an urban scenario is more difficult. For this reason, we consider a few urban scenarios, obtained by simple variations of a starting reference scenario. In particular, we have taken into account a general scenario, constituted by a single horizontal (east-west) road and several intersecting vertical (south-north) roads, whose number is denoted as $N_{\text {road }}^{\mathrm{v}}$. In all considered scenarios, $N_{\text {road }}^{\mathrm{v}} \in\{0,1,2\}$. When $N_{\text {road }}^{\mathrm{v}}=0$ the vehicles can only proceed east or west, as there is no route towards north and south. On the contrary, when $N_{\text {road }}^{\mathrm{v}}>0$ at each junction the vehicles can move towards all four cardinal directions.

Each road has a length equal to $L_{\text {road }}=\ell_{\text {norm }} z$ and is composed by $N_{\text {lane }}$ adjacent lanes: $N_{\text {lane }}-1$ are reserved for the vehicles entering the network (inbound) and the remaining one is reserved for the vehicles exiting the network (outbound). We foresee two types of junctions: (i) the first one is regulated by a roundabout $(\mathrm{R})$ with radius $10 \mathrm{~m}$, whereas (ii) the second one is regulated by traffic lights (TLs), whose number is equal to the number of available directions. During its duty cycle, a TL stays green for $T_{\text {green }}=55 \mathrm{~s}$, red for $T_{\text {red }}=60 \mathrm{~s}$, and amber for $T_{\text {amber }}=5 \mathrm{~s}$. Obviously, the TLs lying in orthogonal roads have an orthogonal duty cycle with respect to those in the horizontal road, under the assumption that the amber and green colors are orthogonal with respect to the red color. Moreover, in the presence of multiple intersections we assume that all TLs in the horizontal road are synchronized. The number of junctions will be denoted as $N_{\text {jun }}$.

The vehicles enter the considered spatial region according to a global (i.e., over all inbound lanes of the scenario at hand) time-domain Poisson process of parameter $\gamma$ (dimension: [veh/s]). Once generated, each vehicle appears, according to a uniform selection, in one of the available inbound lanes. Once a vehicle enters the network, it follows a random itinerary along the available roads, randomly determining its direction in correspondence to each junction. The vehicle generation process stops as soon the number of generated vehicles reaches a pre-fixed value denoted as $N$ (as in the highway scenario). ${ }^{2}$

By construction, the urban scenario is square-shaped with edges of fixed length $L=L_{\text {road }}$. The RSU is always placed at the center of the square region and we define the ROI as the square region centered around the RSU with edges of fixed length $L_{\mathrm{ROI}}=\ell_{\mathrm{ROI}} z$. Despite its square shape, urban scenario cannot be considered as a purely bidimensional scenario, since the positions of the vehicles are still constrained by the road infrastructure, which only includes horizontal and vertical roads. Conversely, if we ignore the relatively small area of the junctions, the horizontal and vertical roads can be considered as quasimonodimensional spaces. This assumption allows one to define the following approximate per-road vehicle linear spatial density:

$$
\rho_{\mathrm{s}} \simeq \frac{N}{\left(1+N_{\mathrm{road}}^{\mathrm{v}}\right) L_{\mathrm{road}}}
$$

where 1 horizontal road and $N_{\text {road }}^{\mathrm{v}}$ vertical roads are considered. The symbol used to denote the per-road vehicle spatial density is the same used in the highway scenario. However, while in the highway scenario the definition of $\rho_{\mathrm{s}}$ given in (1) is exact, in the current case the per-road linear spatial density given by (2) is an approximation. In the special case with $N_{\text {road }}^{\mathrm{v}}=0$, the linear vehicular density on the right-hand side of (2) becomes exact. Similarly to the highway scenarios, in the urban scenarios $\rho_{\mathrm{s}}^{\mathrm{R}}(t)$ denotes the instantaneous average per-road (linear) vehicle spatial density in the ROI, where the average is carried out over all roads - in fact, different roads in the ROI are likely to have, at the same instant, different instantaneous vehicle spatial densities. In other words, $\rho_{\mathrm{s}}^{\mathrm{R}}(t)$ is obtained from (2) by replacing $N$ with $N_{\text {ROI }}(\mathrm{t})$.

We consider eight instances of the above common urban topology reference scenario, by varying the junction type and the values of $N_{\text {road }}^{\mathrm{v}}$ and $N_{\text {jun. }}$ A generic urban scenario instance will be denoted as $\mathrm{X}_{\mathrm{Y}}^{\mathrm{Z}}$, where $\mathrm{X}$ indicates the junction type ( $\mathrm{R}$ or TL), $\mathrm{Y}$ indicates the number of junctions $\left(N_{\text {jun }} \in\{1,2\}\right)$, and $\mathrm{Z}$ denotes the presence of vertical roads - in particular, " $\mathrm{Z}=\mathrm{hv}$ " when $N_{\text {road }}^{\mathrm{v}}>0$, and " $\mathrm{Z}=\mathrm{h}$ " when $N_{\text {road }}^{\mathrm{v}}=0$. The eight considered urban scenario and their main parameters are summarized in Table I. The urban topologies with $N_{\text {road }}^{\mathrm{v}}=N_{\text {jun }}>$ 0 (namely, $\mathrm{TL}_{1}^{\mathrm{hv}}, \mathrm{TL}_{2}^{\mathrm{hv}}, \mathrm{R}_{1}^{\mathrm{hv}}$, and $\mathrm{R}_{2}^{\mathrm{hv}}$ ) are shown in Figure 3 . The scenarios with $N_{\text {road }}^{\mathrm{v}}=0$ can be obtained by simply removing the vertical roads from the topologies shown in Figure 3, still leaving the junctions. Although this may not be realistic from a practical viewpoint (there is no point in using a $\mathrm{R}$ or TL if there is no crossing road), it is meaningful from an information dissemination viewpoint (the propagation of information along a single direction may be meaningful).

\footnotetext{
${ }^{2}$ We remark that, as in the highway scenario, once $N$ cars are generated, they will remain in the scenario till the end of the simulations. In fact once a car exits from an outbound lane it re-enters immediately from an in-bound lane.
} 


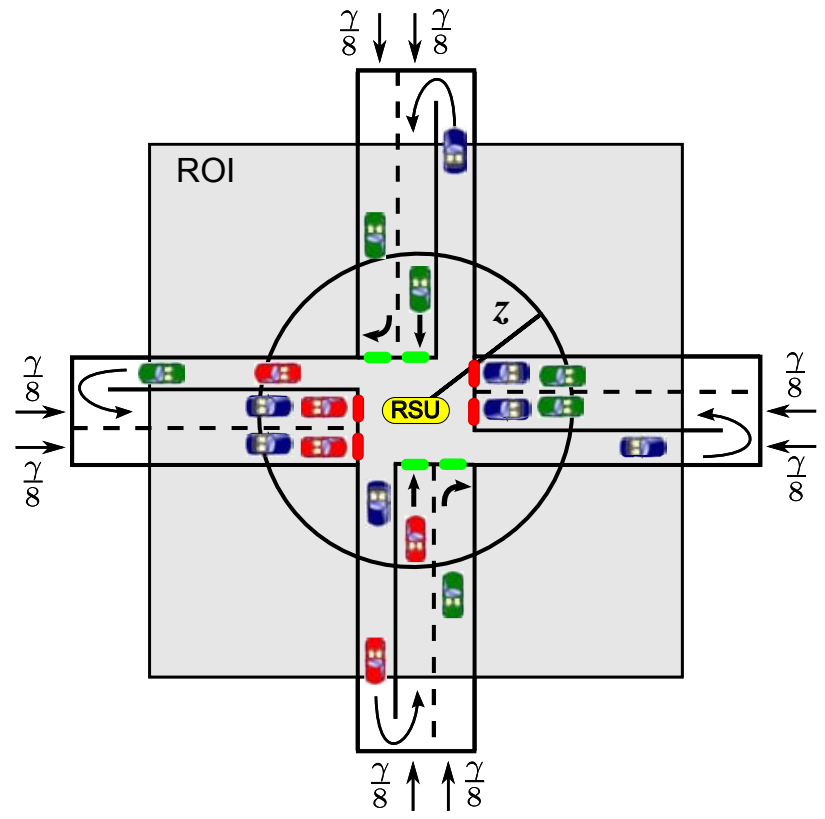

(a)

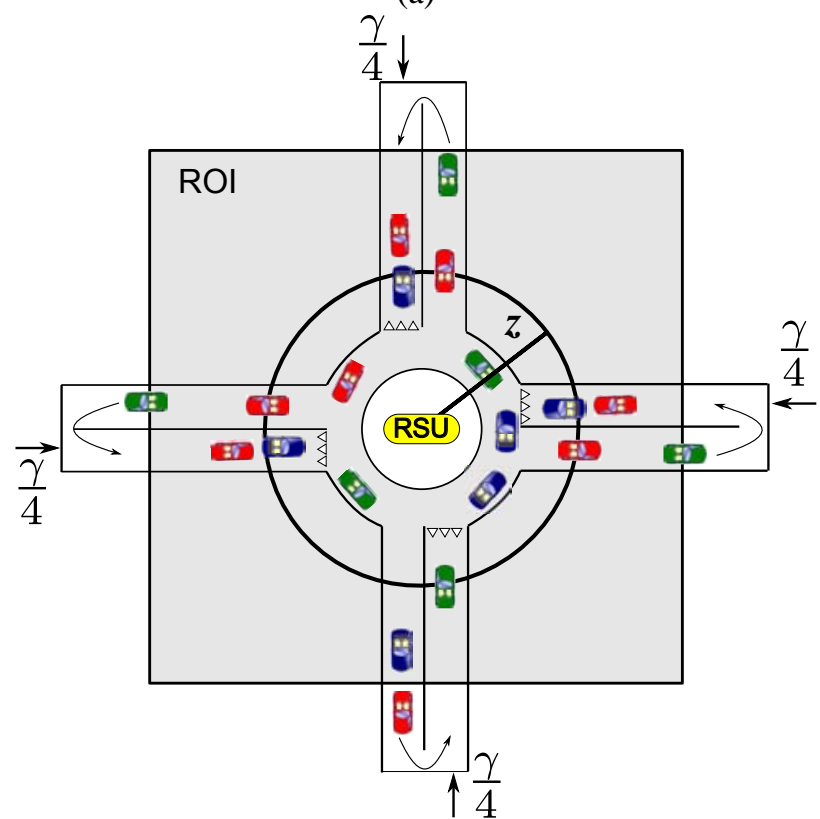

(c)

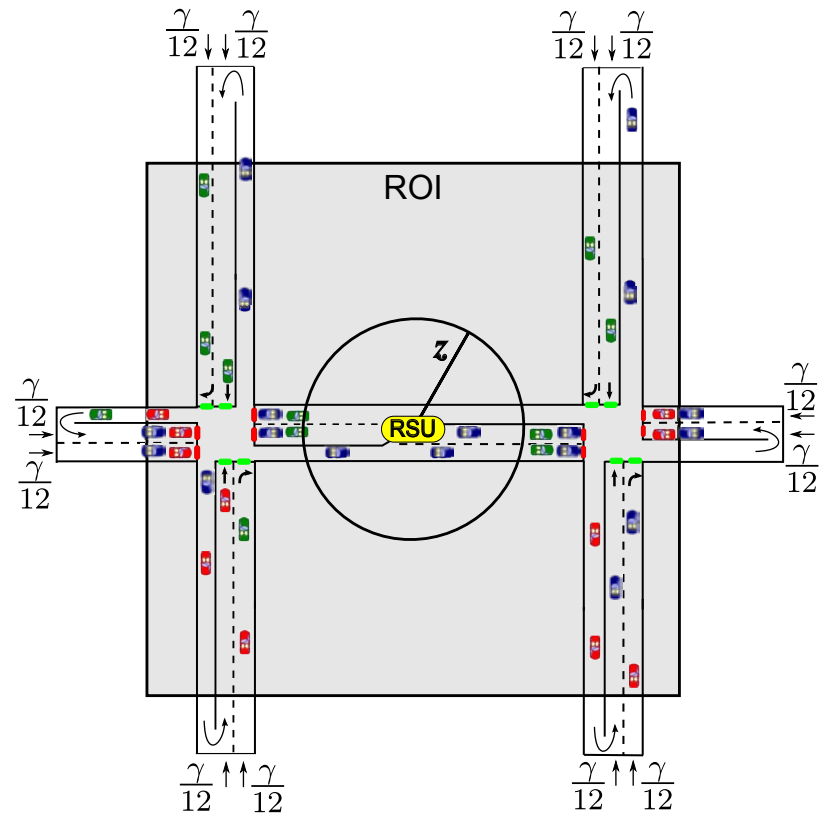

(b)

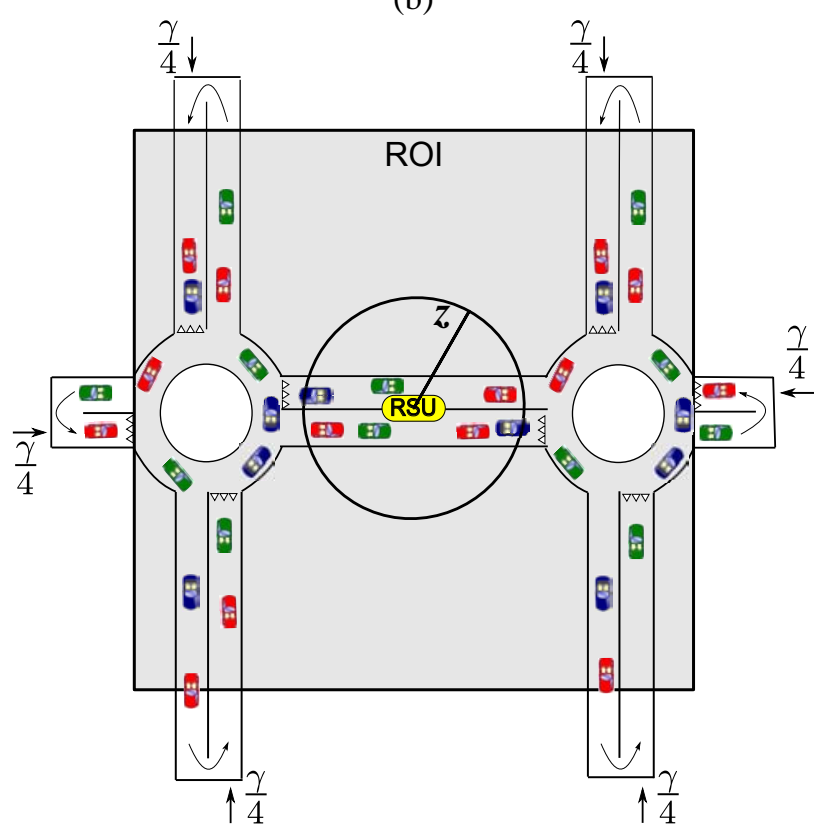

(d)

Figure 3. Urban topologies, with main parameters summarized in Table I, with $N_{\text {road }}^{\mathrm{v}}>0$ : (a) $\mathrm{TL}_{1}^{\mathrm{hv}}$, (b) $\mathrm{TL}_{2}^{\mathrm{hv}}$, (c) $\mathrm{R}_{1}^{\mathrm{hv}}$, and (d) $\mathrm{R}_{2}^{\mathrm{hv}}$.

The approximate definition of per-road vehicular linear spatial density given in (2) is valid regardless of the urban topology at hand, i.e., the type of the horizontal road (with single or double TL or R). However, the dynamic behavior of $\rho_{\mathrm{S}}^{\mathrm{R}}(t)$ can vary significantly in the various cases.

\section{Mobility Models}

In this section, we characterize the mobile highway and urban scenarios introduced in Section II, emphasizing the different mobility characteristics in these topologies. We preliminary remark that a mobile highway scenario will be generated using VanetMobiSim [11], while a mobile urban scenario will be generated using Simulation of
Urban MObility (SUMO) [12]. In fact, the first simulator is very accurate for highway scenarios, whereas the second allows to generate complicated urban topologies in an easier manner. As a by-product of this choice, the comparative results presented here can also be used to reciprocally validate these software applications. VanetMobiSim and SUMO are both open source software able to generate the movement patterns of a certain number of vehicles in a given spatial region and they have several points in common. More specifically, they are based on microscopic mobility models, able to characterize the dynamic behavior of every single vehicle in the network, considering its interactions with other vehicles and with the road signalization. In addition, both products allow to 
TABLE I.

PARAMETERS OF THE CONSIDERED MOBILE URBAN SCENARIOS.

\begin{tabular}{|c|c|c|c|c|}
\hline Scen. & $N_{\text {road }}^{\mathrm{v}}$ & $N_{\text {jun }}$ & Jun. Type & $N_{\text {lane }}$ \\
\hline \hline $\mathrm{TL}_{1}^{\mathrm{h}}$ & 0 & 1 & $\mathrm{TL}$ & 3 \\
\hline $\mathrm{TL}_{2}^{\mathrm{h}}$ & 0 & 2 & $\mathrm{TL}$ & 3 \\
\hline $\mathrm{R}_{1}^{\mathrm{h}}$ & 0 & 1 & $\mathrm{R}$ & 2 \\
\hline $\mathrm{R}_{2}^{\mathrm{h}}$ & 0 & 2 & $\mathrm{R}$ & 2 \\
\hline $\mathrm{TL}_{1}^{\mathrm{hv}}$ & 1 & 1 & $\mathrm{TL}$ & 3 \\
\hline $\mathrm{TL}_{2}^{\mathrm{hv}}$ & 2 & 2 & $\mathrm{TL}$ & 3 \\
\hline $\mathrm{R}_{1}^{\mathrm{hv}}$ & 1 & 1 & $\mathrm{R}$ & 2 \\
\hline $\mathrm{R}_{2}^{\mathrm{hv}}$ & 2 & 2 & $\mathrm{R}$ & 2 \\
\hline
\end{tabular}

generate mobility patterns starting from real maps or by manually defining the road infrastructures by means of graphs.

In all the scenarios considered in this section, $\ell_{\mathrm{ROI}}=8$ and $\ell_{\text {norm }}=10$, with the only exception of the $H_{\text {sta }}$ scenario, where $\ell_{\text {norm }}=8$.

\section{A. Highway Scenarios}

While in the static highway scenario (introduced in Subsection II-B) all vehicles have zero relative speed differences (equivalently, do not move), in the mobile highway scenario (introduced in Subsection II-A), the Intelligent Driver Motion with Lane Changes (IDM-LC) mobility model [13] is used to characterize the mobility of vehicles-recall that the RSU is static.

According to this car-following model, the $i$-th vehicle $(i \in\{1,2, \ldots N\})$ randomly selects a fixed target speed $v_{i}^{\text {target }} \in\left[v^{\min }, v^{\max }\right]$, where $v^{\min }$ and $v^{\max }$ are, respectively, the minimum and maximum speeds. Once its target speed has been selected, the vehicle tries to make its own speed $v_{i}(t)$ reach the target speed. The achievement of this goal is impaired by the road topology and by the presence of other vehicles that accelerate and decelerate. The speed evolution can be analytically characterized by the following equation [14]:

$$
\frac{\mathrm{d} v_{i}(t)}{\mathrm{d} t}=a\left[1-\left(\frac{v_{i}(t)}{v_{i}^{\text {target }}}\right)^{4}-\left(\frac{\delta}{\Delta_{x_{i}}(t)}\right)^{2}\right]
$$

where

$$
\delta \triangleq \Delta_{x_{\min }}+v_{i}(t) T+\frac{v_{i}(t)\left[v_{i}(t)-v_{i+1}(t)\right]}{2 \sqrt{a b}} .
$$

Equation (3) shows that the local acceleration depends on two contributions: (i) the acceleration needed to reach the target speed $v_{i}^{\text {target }}$ and (ii) the deceleration induced by the preceding vehicle. The term $\delta$ defined in (4) determines the desired dynamic distance from the preceding vehicle. This term depends on the following parameters [14]: the safe time headway $(T)$, the maximum acceleration of movement $(a)$, and the comfortable deceleration of movement $(b)$. The values of all parameters in (3) and (4), tuned according to the empirical data presented in [13], are shown in Table II. Moreover, thanks to the integration of the MOBIL lane changing model [4], the IDM-LC mobility model takes also into account possible overtakings between vehicles. In particular, according to
TABLE II.

MAIN PARAMETERS OF THE IDM-LC AND MOBIL MODELS AND THEIR (EMPIRICAL) VALUES.

\begin{tabular}{|c|c|}
\hline Parameter $(i)$ & Value \\
\hline \hline$v^{\min }$ & $20 \mathrm{~m} / \mathrm{s}$ \\
\hline$v^{\max }$ & $40 \mathrm{~m} / \mathrm{s}$ \\
\hline$T$ & $2 \mathrm{~s}$ \\
\hline$a$ & $0.6 \mathrm{~m} / \mathrm{s}^{2}$ \\
\hline$b$ & $0.9 \mathrm{~m} / \mathrm{s}^{2}$ \\
\hline$P$ & 0.5 \\
\hline$b_{\mathrm{safe}}$ & $4 \mathrm{~m} / \mathrm{s}^{2}$ \\
\hline$a_{\mathrm{thr}}$ & $0.2 \mathrm{~m} / \mathrm{s}^{2}$ \\
\hline$L_{\mathrm{v}}$ & $5 \mathrm{~m}$ \\
\hline
\end{tabular}

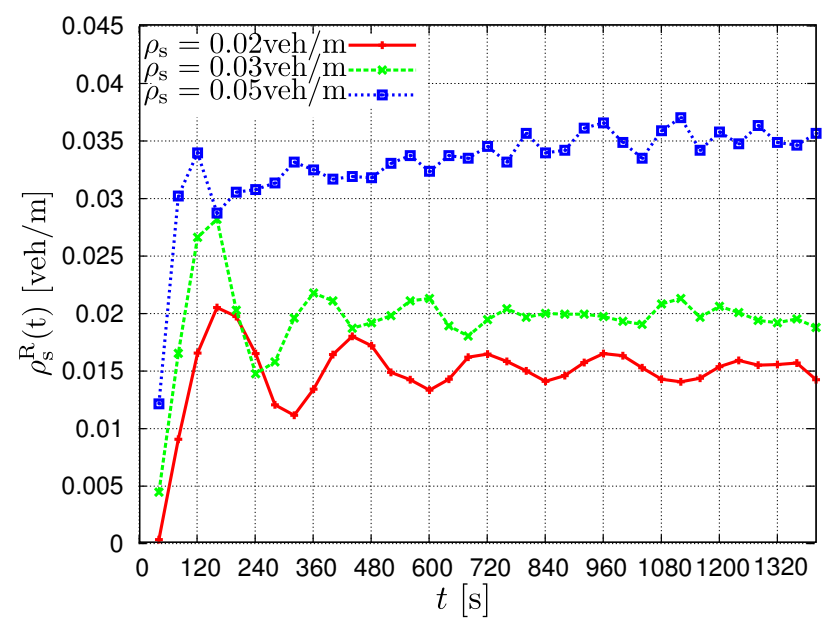

Figure 4. The time evolution of $\rho_{\mathrm{s}}^{\mathrm{R}}(t)$ within the ROI in the $\mathrm{H}_{\mathrm{mob}}$ scenario. Three possible values for $\rho_{\mathrm{S}}$ are considered: $0.02 \mathrm{veh} / \mathrm{m}$, $0.03 \mathrm{veh} / \mathrm{m}$, and $0.05 \mathrm{veh} / \mathrm{m}$. In all cases, $v^{\mathrm{min}}=20 \mathrm{~m} / \mathrm{s}$ and $v^{\max }=40 \mathrm{~m} / \mathrm{s}$.

a game-theoretic approach considered by the MOBIL model, a vehicle (say $i$ ) moves to an adjacent lane if its advantage, in terms of acceleration, is greater than the disadvantage of the preceding vehicle (say $j$ ) in the new lane. This condition can be expressed as

$$
\frac{\mathrm{d} v_{i}^{\prime}(t)}{\mathrm{d} t}-\frac{\mathrm{d} v_{i}(t)}{\mathrm{d} t} \geq P\left(\frac{\mathrm{d} v_{j}(t)}{\mathrm{d} t}-\frac{d v_{j}^{\prime}(t)}{\mathrm{d} t}\right)+a_{\mathrm{thr}}
$$

where the superscript ' refers to the speed values after the potential overtake. The parameter $P$ models the drivers' politeness and the acceleration threshold $a_{\mathrm{thr}}$ prevents lane hopping phenomena in borderline conditions. In order to prevent collisions between the back vehicle $j$ and the overtaking vehicle $i$, there is also the following safety condition on the deceleration of vehicle $j$, based on an arbitrary parameter $b_{\text {safe }}$ :

$$
\frac{d v_{j-i}^{\prime}(t)}{d t} \geq-b_{\text {safe }}
$$

The values of the parameters of the MOBIL model, obtained from experimental data, are also shown in Table II.

In order to validate the highway model, in Figure 4 we show the time evolution of $\rho_{\mathrm{s}}^{\mathrm{R}}(t)$, for $v^{\mathrm{min}}=20 \mathrm{~m} / \mathrm{s}$ and $v^{\max }=40 \mathrm{~m} / \mathrm{s}$. Three values of $\rho_{\mathrm{s}}$ (namely, $0.2 \mathrm{veh} / \mathrm{m}$, $0.3 \mathrm{veh} / \mathrm{m}$, and $0.5 \mathrm{veh} / \mathrm{m}$ ) are considered. The results in Figure 4 show that, regardless of the value of $\rho_{\mathrm{s}}$, after an 
TABLE III.

VALUES OF THE PARAMETERS USED BY SUMO FOR THE GENERATION OF MOBILE URBAN SCENARIOS.

\begin{tabular}{|c|c|}
\hline Parameter $(i)$ & Value \\
\hline \hline$v^{\mathrm{max}}$ & $20 \mathrm{~m} / \mathrm{s}$ \\
\hline$a$ & $0.8 \mathrm{~m} / \mathrm{s}^{2}$ \\
\hline$b$ & $4.5 \mathrm{~m} / \mathrm{s}^{2}$ \\
\hline$\sigma$ & 0.5 \\
\hline$\tau$ & $1 \mathrm{~s}$ \\
\hline$L_{\mathrm{v}}$ & $5 \mathrm{~m}$ \\
\hline
\end{tabular}

initial transient period, $\rho_{\mathrm{s}}^{\mathrm{R}}(t)$ finally converges to a stationary value. This guarantees that the considered mobile scenario can effectively model a stationary highway.

\section{B. Urban Scenarios}

The urban scenarios are generated using SUMO [12], [15], a microscopic road traffic simulator that allows to create a scenario by converting an existing map or, alternatively, by using an external tool (such as NETGEN or NETCONVERT). SUMO uses a car-following dynamic model largely based on the work in [16] (this model will be denoted as KWG) and, therefore, different from the IDM-LC model used in VanetMobiSim. Since the KWG and the IDM-LC models are similar, for the sake of conciseness we omit the analytical details of the KWG model, which can be found in [16]. We only point out that the relevant parameters of the KWG model are: the maximum vehicle speed $\left(v^{\max }\right)$; the maximum acceleration $(a)$; the maximum deceleration $(b)$; the driver's reaction time, denoted as $\tau$ (dimension: [s]); and the dawdling parameter, denoted as $\sigma$ (adimensional), belonging to the interval $[0,1]$. The values of the relevant parameters of the KWG mobility model generated with SUMO are summarized in Table III. We remark that with respect to the IDM-LC model adopted in the $\mathrm{H}_{\text {mob }}$ scenario (Table II), the value of $v^{\max }$ is significantly smaller.

In Figure 5, the time evolution of the instantaneous average per-road (linear) vehicle spatial density (in the ROI) is analyzed in the four urban scenarios (with vertical crossing roads) considered in Figure 3: (a) $\mathrm{TL}_{1}^{\text {hv }}$, (b) $\mathrm{TL}_{2}^{\text {hv }}$, (c) $R_{1}^{\text {hv }}$, and (d) $R_{2}^{\text {hv }}$. In each case, various values of the average per-road vehicle spatial density $\rho_{\mathrm{s}}$ (namely, $0.05 \mathrm{veh} / \mathrm{m}, 0.1 \mathrm{veh} / \mathrm{m}, 0.15 \mathrm{veh} / \mathrm{m}$, and $0.2 \mathrm{veh} / \mathrm{m}$ ) are considered. By observing the results in Figure 5, the following comments can be carried out.

- According to the results in Figure 5 (a) and Figure $5(\mathrm{~b})$, in the scenarios with $T L s \rho_{\mathrm{s}}^{\mathrm{R}}(t)$, after an initial transient, exhibits a cycle-stationary behavior, with period roughly equal to $T_{\text {green }}+T_{\text {red }}+T_{\text {amber }}=$ $120 \mathrm{~s}$. The cycle-stationary nature of $\rho_{\mathrm{s}}^{\mathrm{R}}(t)$ is especially evident in $\mathrm{TL}_{1}^{\mathrm{hv}}$ scenario (case (a)). Note that the average (over time) value of $\rho_{\mathrm{s}}^{\mathrm{R}}(t)$ in the $\mathrm{TL}_{2}^{\mathrm{hv}}$ scenario (case (b)) is slightly smaller than the "target value" $\rho_{\mathrm{s}}$ in the case with $\rho_{\mathrm{s}}=0.2 \mathrm{veh} / \mathrm{m}$. This is mostly due to the fact that the ROI is placed between the two traffic junctions, as shown in Figure 3 (b).

- In the scenarios with Rs, from the results in Figure 5 (c) and Figure 5 (d) one can conclude that
$\rho_{\mathrm{s}}^{\mathrm{R}}(t)$, after the initial transient, tends to a stationary condition. At regime, there are still minor oscillations, which do not seem periodic. Unlike the scenarios with TLs, in both scenarios $\mathrm{R}_{1}^{\mathrm{hv}}$ and $\mathrm{R}_{2}^{\text {hv }}$, the steady-state average (over time) value of $\rho_{\mathrm{s}}^{\mathrm{R}}(t)$ tends to be greater than the "target" value $\rho_{\mathrm{s}}$, especially for high values of $\rho_{\mathrm{s}}$. This happens because a $\mathrm{R}$ junction has a smaller vehicle capacity of a TL junctions.

\section{Comparison Between Highway and Urban Scenarios}

First of all, by comparing Figure 4 and Figure 5 one can observe that with both SUMO and VanetMobiSim it is not straightforward to obtain a "target" value of $\rho_{\mathrm{s}}$. However, SUMO seems to guarantee a more refined control on the vehicle spatial density than VanetMobiSim. A better control could be obtained by considering wider areas and a larger number of vehicles, but this would lead to an explosion of the simulation time.

Secondly, due to the significant differences between urban and highway scenarios, we have chosen different values of speeds and vehicle spatial densities. In order to emphasize this difference, in Figure 6 (a) we show the dispersion diagrams of the instantaneous per-road vehicle spatial density $\rho_{\mathrm{s}}^{\mathrm{R}}(t)$ with respect to the node speed $v(t)$, in the $\mathrm{H}_{\mathrm{mob}}$ and $\mathrm{TL}_{1}^{\text {hv }}$ scenarios. In the $\mathrm{H}_{\text {mob }}$ scenarios, three values of $\rho_{\mathrm{s}}(0.02 \mathrm{veh} / \mathrm{m}, 0.03 \mathrm{veh} / \mathrm{m}$, and $0.05 \mathrm{veh} / \mathrm{m}$ ) are considered, whereas in $\mathrm{TL}_{1}^{\mathrm{hv}}$ scenario four values of $\rho_{\mathrm{s}}(0.05 \mathrm{veh} / \mathrm{m}, 0.1 \mathrm{veh} / \mathrm{m}, 0.15 \mathrm{veh} / \mathrm{m}$, and $0.2 \mathrm{veh} / \mathrm{m}$ ) are considered. In Figure 6 (b), an enlarged version of the dispersion diagrams of the $\mathrm{H}_{\text {mob }}$ scenarios of Figure 6 (a) are shown. For each specific scenario, i.e., dispersion diagram, each point (i.e., the pair $\left.\left(\rho_{\mathrm{s}}^{\mathrm{R}}(t), v(t)\right)\right)$ corresponds to a specific time instant of the simulation. From the results in Figure 6, one can observe that in the $\mathrm{TL}_{1}^{\mathrm{hv}}$ scenarios the dispersion diagrams tend to aggregate around the average density-speed pair. Moreover-as expected-in all cases the average speed is a decreasing function of the average vehicle spatial density. Finally, one can observe that the average vehicle speeds are generally lower than the maximum speeds set in Table II and Table III. In the $\mathrm{TL}_{1}^{\mathrm{hv}}$ scenario, this behavior can be easily justified by the presence of the junctions. On the other hand, in the $\mathrm{H}_{\text {mob }}$ scenario this can be motivated by the fact that the vehicles do not encounter a sufficiently long free space to reach their target speed. However, the maximum speed value (around $30 \mathrm{~m} / \mathrm{s}$ ) observed in $\mathrm{H}_{\text {mob }}$ scenario with $\rho_{\mathrm{s}}=0.02 \mathrm{veh} / \mathrm{m}$ is realistic for a stationary highway in most of the western countries.

Finally, in Figure 7 we show the dispersion diagrams $\left(\rho_{\mathrm{s}}^{\mathrm{R}}(t), v(t)\right)$ relative to the horizontal road of the $\mathrm{TL}_{2}^{\mathrm{hv}}$ scenario, considering the four values of $\rho_{\mathrm{s}}$ already considered in Figure 6 (a) for the $\mathrm{TL}_{1}^{\text {hv }}$ scenario. In this case, the ROI, placed between two junctions, encompasses both intersections (with TLs) and the horizontal road between them. Therefore, the mobility in the horizontal road between the traffic junctions is influenced by both TL plants, thus yielding to a much higher speed variance 


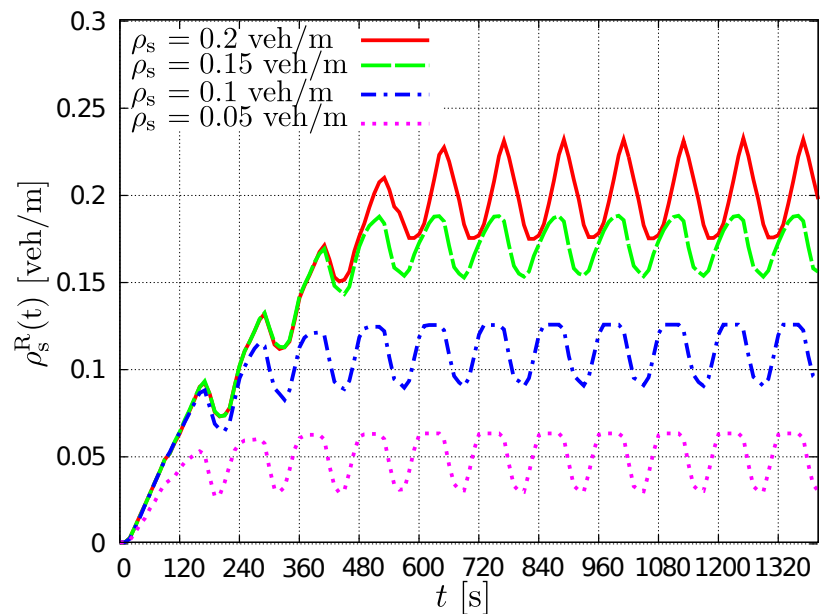

(a)

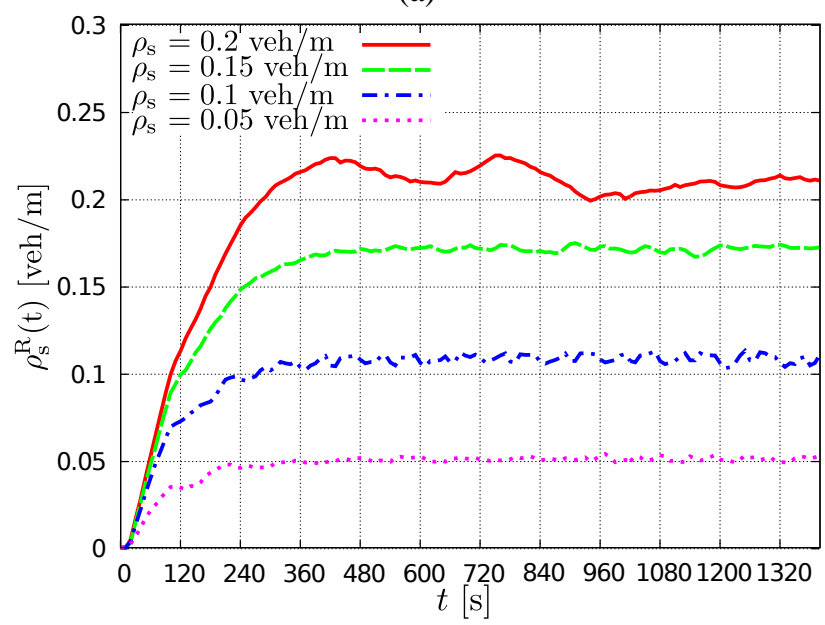

(c)

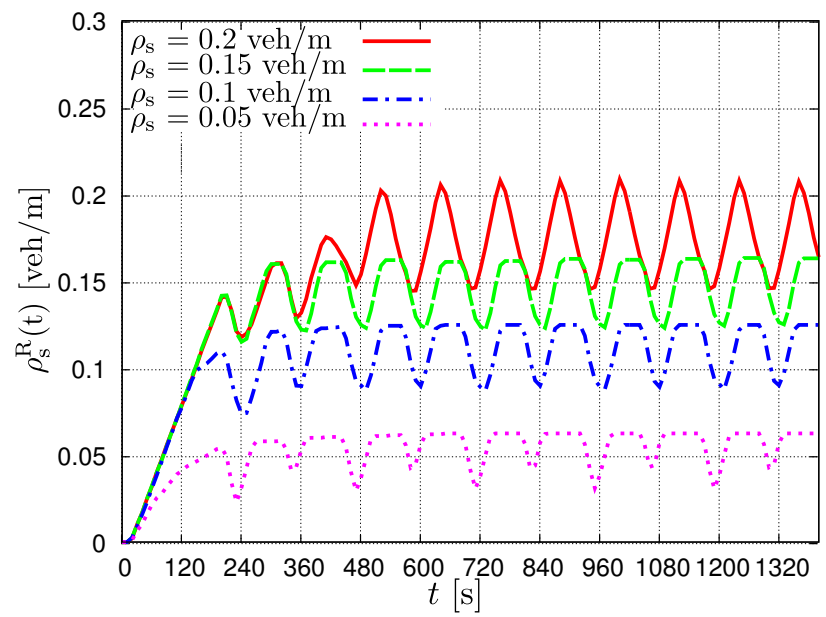

(b)

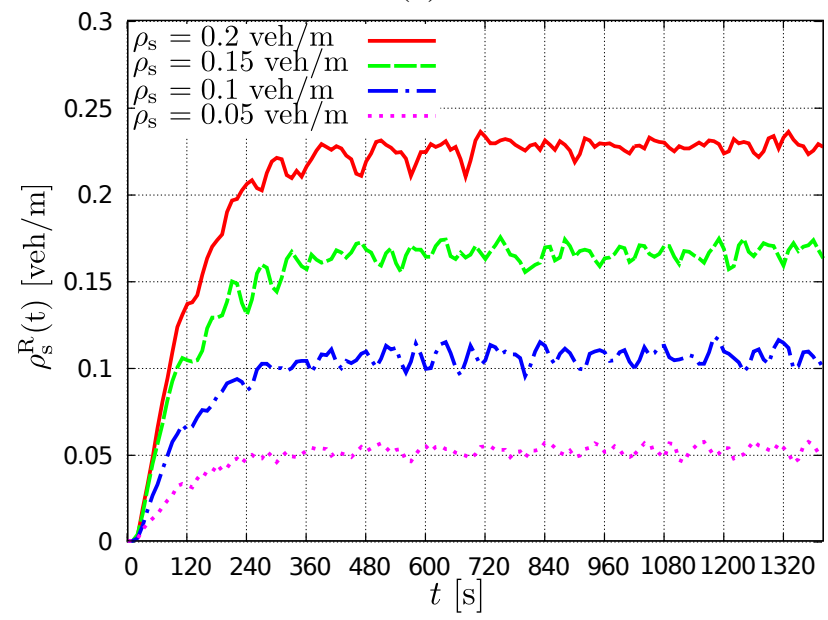

(d)

Figure 5. Average instantaneous per-road (linear) vehicle spatial density (in the ROI) in the four urban scenarios considered in Figure 3: (a) TL ${ }_{1}^{\text {hv }}$, (b) $T L_{2}^{\text {hv }}$, (c) $R_{1}^{\text {hv }}$, and (d) $R_{2}^{\text {hv }}$. In each case, various values of $\rho_{\mathrm{s}}$ are considered.

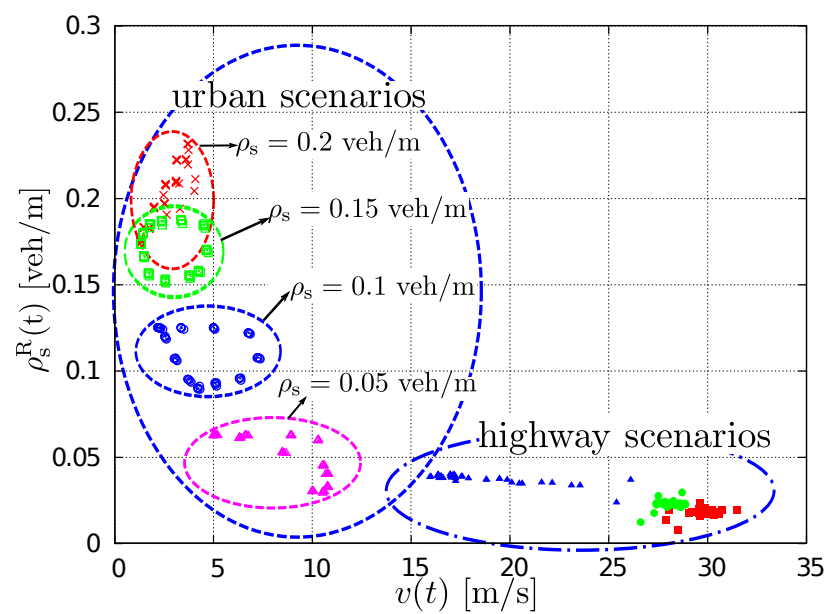

(a)

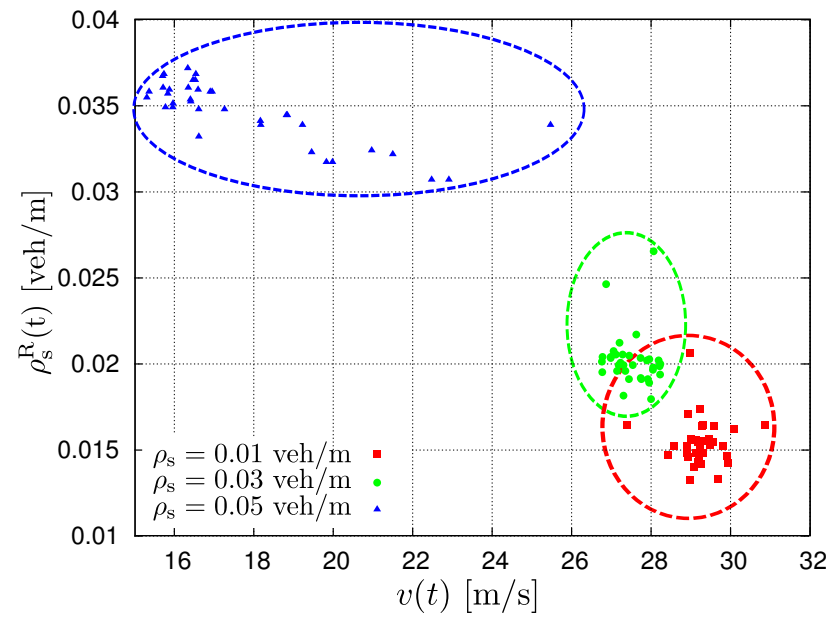

(b)

Figure 6. Average instantaneous per-road (linear) vehicle spatial density (in the ROI) $\left(\rho_{\mathrm{S}}^{\mathrm{R}}(t)\right)$ as a function of the average speed $(v(t))$, parametrized with respect to the time $t$, considering various values of $\rho_{\mathrm{s}}$. In (a) there is a comparison between $\mathrm{H}_{\mathrm{mob}}$ and $\mathrm{TL}_{1}^{\text {hv }}$ scenarios, while in (b) there is an enlargement of the $\mathrm{H}_{\text {mob }}$ scenario of (a).

with respect to the average (with respect to all roads in the ROI) behavior shown in Figure 6 (a).

\section{IRRESPONSIBLE FORWARDING IN VANETS}

\section{A. Irresponsible Forwarding}

The IF protocol was originally introduced for ideal (collision-free) static linear networks [7] and then ex- 


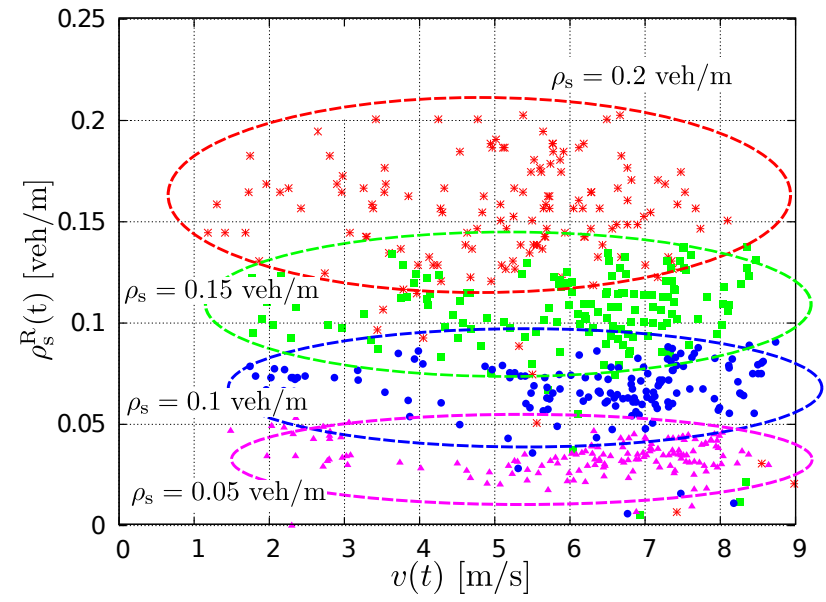

Figure 7. Average instantaneous vehicle spatial density (in the horizontal road of the ROI) $\left(\rho_{\mathrm{s}}^{\mathrm{R}}(t)\right)$ as a function of the average speed $(v(t))$, parametrized with respect to the time $t$, considering various values of $\rho_{\mathrm{S}}$.

tended to static IEEE 802.11 linear networks [8]. For the sake of conciseness, we describe the IF protocol referring to the portion of length $L$ of a single lane of a highway, as shown in Figure 2. Let us consider a vehicle, at a generic distance $d$ from the source (RSU), within the transmission range of the source (i.e., $d<z$ ). According to the idea of the IF protocol, the vehicle should rebroadcast the packet only if the probability of finding another vehicle in the consecutive interval of length $z-d$ is low: otherwise, it should not. More specifically, when a vehicle receives a packet at time $t$, it compares its position with that of the transmitter and computes its rebroadcast probability $p(t)$ as follows:

$$
p(t)=\exp \left\{-\frac{\rho_{\mathrm{s}}^{\mathrm{v}}(t)(z-d)}{c}\right\}
$$

where $c \geq 1$ is a tunable parameter which can be selected to "shape" the probability of rebroadcasting (as a function of $d$ ) - the higher the value of $c$, the higher the probability of rebroadcasting at any position $d$-and $\rho_{\mathrm{s}}^{\mathrm{v}}(t)$ is the local vehicle spatial density, evaluated by each vehicle, independently from the other vehicles, at time $t$. The local spatial density $\rho_{\mathrm{s}}^{\mathrm{v}}(t)$ can be significantly different from the per-road vehicle spatial $\rho_{\mathrm{s}}^{\mathrm{R}}(t)$, but they usually have the same order of magnitude. We remark that the IF protocol acts on a per-packet basis and does not keep memory of past forwarding decisions.

\section{B. Performance Metrics}

The performance analysis of IF in the VANET scenarios introduced in the previous sections will be carried out considering both per-packet and per-flow metrics. The former type of metrics is suitable to validate the performance of applications, such as safety-related applications, where a single broadcast packet needs to be disseminated. The latter type of metrics, instead, is suitable to evaluate the performance of applications, such the content distribution applications, where a significant amount of information needs to be distributed using a flow of hundreds of broadcast packets. In all cases, these metrics will be evaluated through simulations.

Two per-packet performance metrics are considered: (i) the Reachability (RE), originally introduced in [17] and defined as the fraction of nodes that receive the source packet among the set of all reachable nodes; (ii) the average end-to-end delay at the $i$-th hop, denoted as $D_{i}$ (dimension: [s]) and computed as follows. In each simulation run, we average over the delays experienced by all vehicles which receive the packet at the $i$-th hop in order to derive the average end-to-end delay at the $i$ th hop. The average end-to-end delay at the $i$-th hop is evaluated as:

$$
D_{i}=\sum_{j=1}^{i} D_{\mathrm{hop}-j}
$$

where $D_{\text {hop- } j}$ is the delay of the $j$-th hop along the multihop path.

A single per-flow performance metric is considered, namely the instantaneous throughput. This metric, associated to a specific vehicle, is defined as

$$
S^{(i)}(t) \triangleq \frac{N_{\mathrm{p}-\mathrm{rx}}^{(i)}(t)}{N_{\mathrm{p}}}
$$

where $N_{\mathrm{p}-\mathrm{rx}}^{(i)}(t)$ is the number of received packets till time $t$ by the considered $i$-th vehicle-assuming that the simulation run starts at time 0 - and $N_{\mathrm{p}}$ is the number of packets of the flow.

\section{Simulation Setup}

We consider the wireless communication protocol stack defined by the ad-hoc IEEE 802.11 standard [18]. Due to the broadcast nature of the communications, the RequestTo-Send/Clear-To-Send (RTS/CTS) mechanism foreseen by the standard cannot be exploited, and correct packet reception cannot be acknowledged. Therefore, the packets are never retransmitted and they experience a single backoff whose length is randomly selected in the set $\left\{0,1, \ldots, \mathrm{CW}_{\min }\right\}$, where $\mathrm{CW}_{\min }=31$ as established by the IEEE $802.11 \mathrm{~b}$ standard. The IF protocol is "inserted" on top of the IEEE 802.11 b model present in Network Simulator 2 (ns-2.31 [19]), after fixing some bugs present in this release. The data rate from each node is set to $1 \mathrm{Mb} / \mathrm{s}$ and the packets are generated according to a Poisson transmission distribution with parameter $\lambda$ (dimension: [pck/s]). Since this work does not focus on physical layer issues, we adopt a simple Friis free-space propagation model. While this is realistic for highway scenarios, it applies to urban scenarios without significant buildings around the roads. In other words, our analysis of urban scenarios aims at investigating the impact of traffic control (through TLs or Rs). The extension of our analysis to urban scenarios with tall buildings around the streets (e.g., in Manhattan, New York), which create wireless "waveguides," would require careful modeling of the propagation conditions. 
In the case of per-packet (Section V) and per-flow (Section VI) performance analysis, the packet size (PS) will be fixed to either 100 bytes or 1000 bytes. The considered performance metrics will be evaluated by averaging over 100 simulation runs.

- In the per-packet analysis, a single simulation run corresponds to a randomly generated VANET scenario (nodes' generation and associated mobility patterns) and to the transmission of $N_{\mathrm{p}}=1000$ packets by the RSU. At the end of each simulation run, the delay and reachability are obtained by averaging over the delays and reachabilities of all vehicles. The final results of the simulation are then obtained by averaging over the results of all simulation runs.

- In the per-flow analysis, in each simulation run we consider the same randomly generated VANET scenario (nodes' generation and mobility pattern) in other words, the scenario generated at the first run is kept unchanged in the following 99 runs. However, run by run the transmission scheduling of the $N_{\mathrm{p}}=$ 1000 packets by the RSU varies, according to their generations. At the end of each simulation run, we evaluate the throughput experienced by all vehicles within the ROI. Finally, after all simulation runs, we evaluate the average throughput experienced by each vehicle. Note that the fact of keeping the VANET scenario fixed allows to reevaluate the throughput of each vehicle, thus emphasizing the role of the specific path followed by each vehicle.

In both per-packet and per-flow performance analysis, simulations will be carried out considering the (highway and urban) scenarios described in Section II, using the mobility models introduced and characterized in Section III-this is possible since both VanetMobiSim and SUMO are able to generate mobility traces compatible with ns-2. Therefore, the only information source in the network is the RSU, always placed at the center of the ROI.

As considered in Section II for the characterization of the mobility models, in Section V and Section VI the performance analysis of the IF protocol will be carried out considering $\ell_{\mathrm{ROI}}=8$ and $\ell_{\text {norm }}=10$ in all scenarios but the $\mathrm{H}_{\text {sta }}$ scenario, where $\ell_{\text {norm }}=8$. In all cases, the shaping factor $c$ of the IF protocol in (7) will be set to 5 .

In order to obtain fair results, in all the comparisons carried out in Section V and Section VI, we fix the same target per-road density $\rho_{\mathrm{s}}$ in every scenario. As a consequence of that, the number of nodes $N$ varies from scenario to scenario. In particular, if we fix a target perroad density $\rho_{\mathrm{s}}$, from the equation (2) we obtain that the overall number of nodes is given by:

$$
N=\rho_{\mathrm{s}} L_{\text {road }}\left(1+N_{\text {road }}^{\mathrm{v}}\right)
$$

where $\rho_{\mathrm{S}} L_{\mathrm{road}}$ is the number of per-road nodes.

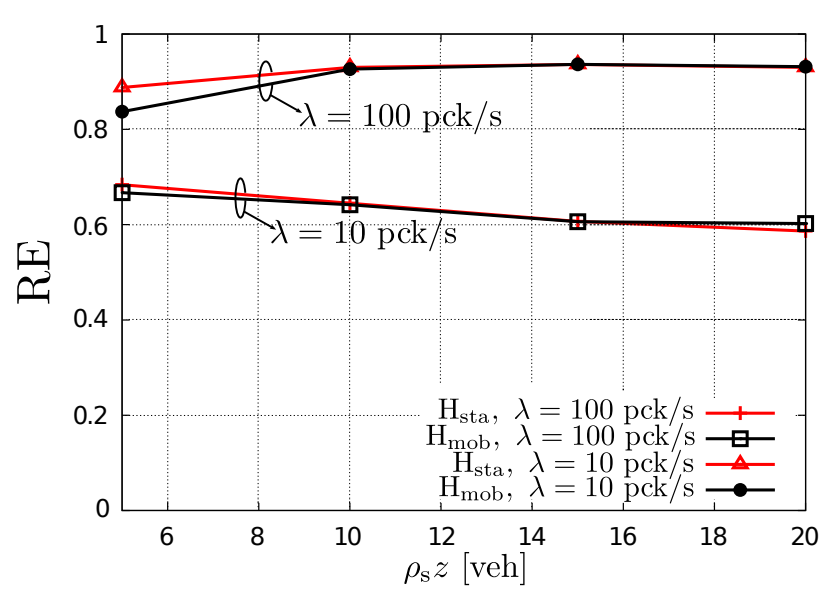

Figure 8. RE, as a function of $\rho_{\mathrm{s}} z$, in the $\mathrm{H}_{\mathrm{sta}}$ and the $\mathrm{H}_{\mathrm{mob}}$ scenarios. Two values of $\lambda$ are considered, namely, 10 and $100 \mathrm{pck} / \mathrm{s}$.

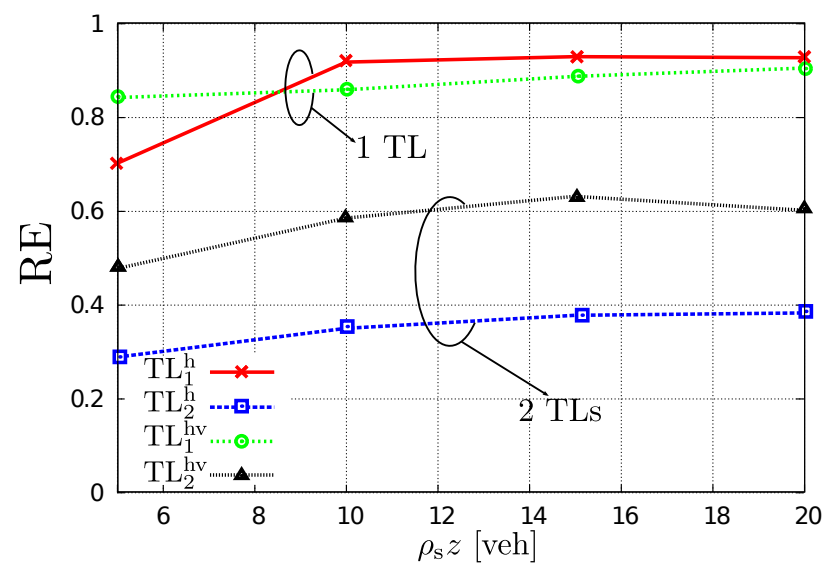

Figure 9. RE as a function of $\rho_{\mathrm{s}} z$, in the $\mathrm{TL}_{1}^{\mathrm{hv}}, \mathrm{TL}_{2}^{\mathrm{hv}}, \mathrm{TL}_{1}^{\mathrm{h}}$, and $\mathrm{TL}_{2}^{\mathrm{h}}$ urban scenarios. In all cases $\lambda=10 \mathrm{pck} / \mathrm{s}$.

\section{Per-PACKet Performance of IF}

\section{A. Highway Scenarios}

Although the static $\left(\mathrm{H}_{\text {sta }}\right)$ and mobile $\left(\mathrm{H}_{\text {mob }}\right)$ highway scenarios have the same road topology, they are characterized by very different dynamic conditions. A direct comparison of the performance of the IF protocol in these two cases allows to clearly understand the impact of dynamic conditions in a highway scenario. In Figure 8, the $\mathrm{RE}$ is shown as function of $\rho_{\mathrm{s}} z$, considering two values of $\lambda(10 \mathrm{pck} / \mathrm{s}$ and $100 \mathrm{pck} / \mathrm{s})$. From the results in Figure 8 , it emerges clearly that in a stationary highway scenario the behavior of a broadcast protocol such as IF is not significantly affected by the dynamic state of the vehicle. In fact, for a given value of $\lambda$, the performance experienced in a mobile scenario is basically identical (but for minor differences at low values of $\left.\rho_{\mathrm{s}} z\right)$ to that in a static scenario, because the mobility has no effect on the performance of IF, due to the stateless nature of the broadcast protocol.

\section{B. Urban Scenarios}

In Figure 9, the RE obtained in urban scenarios with TLs is shown as a function of $\rho_{\mathrm{s}} z$. All four urban 
scenarios with TLs shown in Table I are considered. In all cases, $\lambda=10 \mathrm{pck} / \mathrm{s}$. From the results in Figure 9, it can be concluded that the number of TLs has a strong impact on the RE. In fact, the performance with one TL is better, regardless of the presence of an intersecting vertical road, than the performance with two TLs. Depending on the number of TLs, the following comments can be carried out.

- In the presence of one $T L$, it can be observed that in the $\mathrm{TL}_{1}^{\mathrm{hv}}$ scenario (there is an intersecting vertical road) the RE has roughly the same behavior (slightly increasing with respect to $\rho_{\mathrm{s}} z$ ) of that in the highway scenarios. This can be easily justified. In fact, when the TL in one of the two intersecting roads is green, the vehicle configuration along this road is quite similar to the highway scenario, with the exception of a lower average speed and a smaller number of lanes: therefore, the behavior is similar. Conversely, when the TLs of this road become red (the other road has green TLs, i.e., it behaves as described before), there are two local clusters of highly connected static vehicles, thus leading to a higher probability of successful packet forwarding.

In the $\mathrm{TL}_{1}^{\mathrm{h}}$ scenario (there is no intersecting vertical road), the RE is lower than that of the $\mathrm{TL}_{1}^{\mathrm{hv}}$ scenario for small values of $\rho_{\mathrm{s}} z$ and then becomes higher for increasing values of $\rho_{\mathrm{s}} z$. Since there is no intersecting vertical road, connectivity is completely "delegated" to the horizontal road. When the TLs are green, if $\rho_{\mathrm{s}} z$ is too small the VANET becomes sparse, connectivity is lost, and the RE is low. For sufficiently large values of $\rho_{\mathrm{s}} z$, even when the TLs are green, the moving cars are sufficiently dense and packet forwarding is guaranteed. In particular, the RE becomes slightly higher than in the $\mathrm{TL}_{1}^{\mathrm{hv}}$ scenario, as there is a reduced number of forwarding collisions around the junction-recall that in the $\mathrm{TL}_{1}^{\mathrm{hv}}$ case, when the TLs along the horizontal road are green there are clusters of stopped vehicles in the vertical road.

- In presence of two TLs, the RE exhibits a radically different behavior with respect to the previous case and significantly lower values. Recalling the structure of the ROI shown in Figure 3 (b), the results in Figure 9 can be interpreted as follows. In the $\mathrm{TL}_{2}^{\mathrm{h}}$ scenario (there are no intersecting vertical roads), when both TLs (along the horizontal road) are red, there are two separated clusters of connected static vehicles, relatively distant from the RSU. Therefore if a packet cannot reach one of these clusters, say the leftmost one, all the vehicles at the left side of the network will not receive the packets transmitted by the RSU.

Unlike the case with one TL, in the scenarios with two TLs the presence of intersecting vertical roads has a very beneficial impact: in Figure 9, the RE in the $\mathrm{TL}_{2}^{\mathrm{hv}}$ scenario is significantly higher, regardless of the value of $\rho_{\mathrm{s}} z$, than in the $\mathrm{TL}_{2}^{\mathrm{h}}$ scenario.

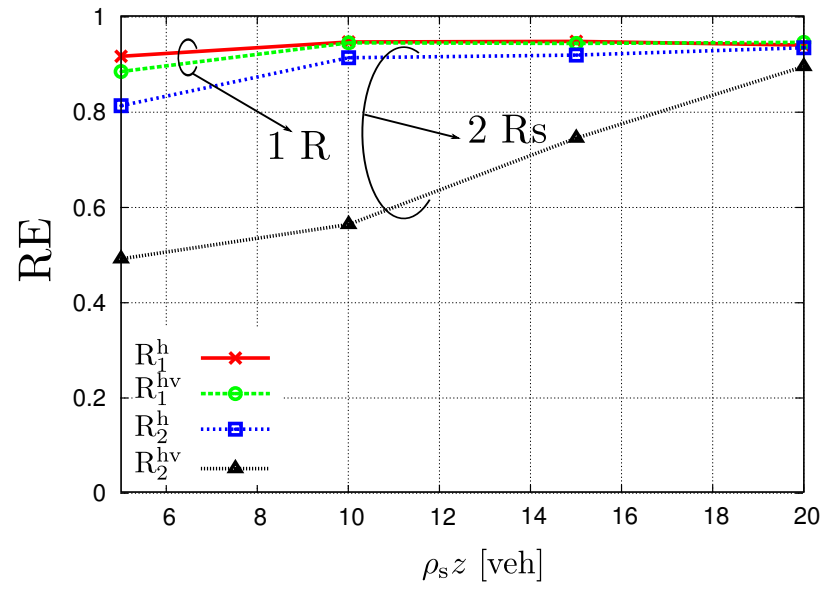

Figure 10. RE, as a function of $\rho_{\mathrm{s}} z$, in the $\mathrm{R}_{1}^{\mathrm{hv}}, \mathrm{R}_{2}^{\mathrm{hv}}, \mathrm{R}_{1}^{\mathrm{h}}$, and $\mathrm{R}_{2}^{\mathrm{h}}$ urban scenarios. In all cases $\lambda=10 \mathrm{pck} / \mathrm{s}$.

In fact, the presence of vertical intersecting roads guarantees that even if the TLs along the horizontal road are red, there will be some vehicles, coming from the vertical roads, which turn towards the RSU, thus guaranteeing connectivity in the street segment, between the two junctions, in the middle of which the RSU is placed.

In Figure 10, the RE obtained in urban scenarios with Rs is shown as a function of $\rho_{\mathrm{s}} z$-this figure is the equivalent of Figure 9, with the same settings, but for the replacement of TLs with Rs. A very different behavior, with respect to the urban scenarios with TLs can be observed.

- In the scenarios without vertical crossing roads $\left(\mathrm{R}_{1}^{\mathrm{h}}\right.$ and $\mathrm{R}_{2}^{\mathrm{h}}$ ), the $\mathrm{RE}$ is very high, regardless of the number of Rs. In other words, the number of roundabouts has no impact. In fact, even in very dense scenarios (large values of $\rho_{\mathrm{s}} z$ ), the roundabouts make the traffic along the horizontal road very fluid, so that the VANETs are almost always connected and packet forwarding is effective.

- In the scenarios with vertical crossing roads $\left(\mathrm{R}_{1}^{\mathrm{hv}}\right.$ and $R_{2}^{\text {hv }}$ ), the $R E$ is high only in the presence of a single $\mathrm{R}\left(\mathrm{R}_{1}^{\mathrm{hv}}\right)$ - for $\rho_{\mathrm{s}} z \geq 10$, the RE is almost the same of that in the scenarios with no crossing roads. Unlike the $\mathrm{TL}_{2}^{\mathrm{hv}}$ scenario, in the $\mathrm{R}_{2}^{\mathrm{hv}}$ (two vertical crossing roads), the $\mathrm{RE}$ is low for small values of $\rho_{\mathrm{s}} z$, and reaches a high value (around 0.8 ) only for very large values of $\rho_{\mathrm{s}} z$. In other words, it turns out that in the case with two consecutive Rs and low vehicle spatial density, the Rs tend to reduce the vehicle flow along the horizontal road (this does not happen in the $\mathrm{TL}_{2}^{\mathrm{h}}$ scenario), making the VANET around the RSU disconnected.

In Figure 11, we analyze the delay, as a function of the hop number, in the scenarios (a) with TLs and (b) Rs. The obtained results (with the slight exception of $\mathrm{TL}_{2}^{\mathrm{hv}}$ in Figure 11 (a)) show that in all scenarios the delay is basically the same till the 5-th hop. Since $\ell_{\mathrm{ROI}}=8$ (as stated at the end of Subsection IV-C) and $z=100 \mathrm{~m}$, 


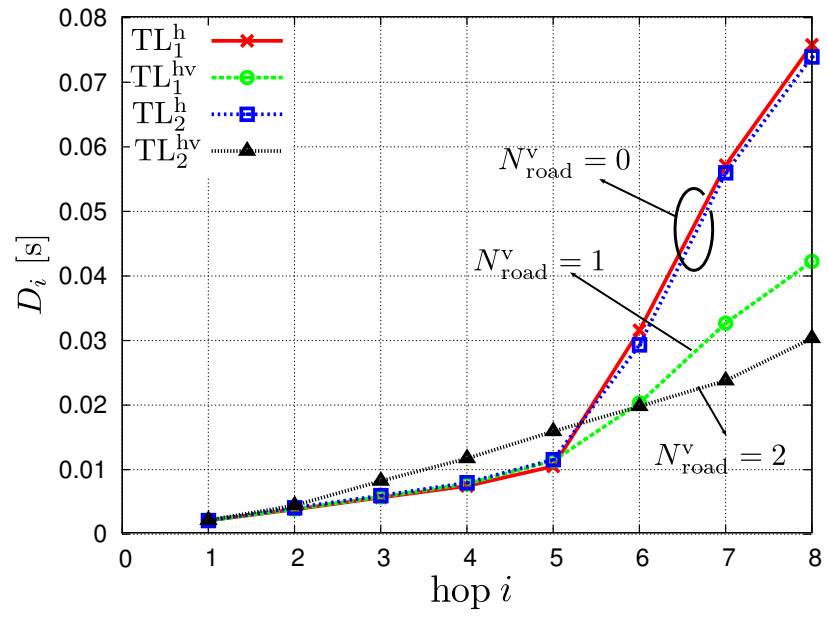

(a)

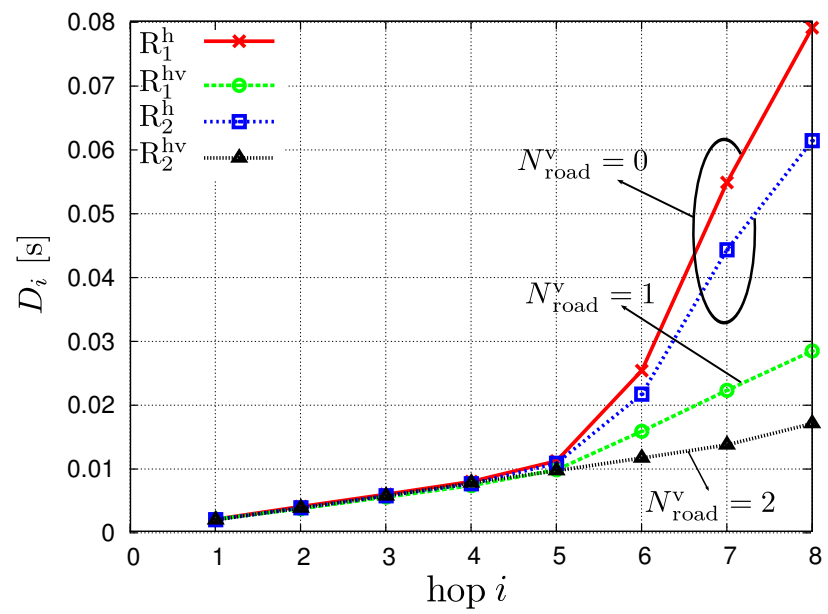

(b)

Figure 11. Delay, as a function of the hop index, in scenarios (a) with TLs and (b) with Rs. In all cases, $\lambda=10$ pck/s, and $\rho_{\mathrm{s}} z=20$ veh.

the side of the square ROI is $\ell_{\mathrm{ROI}} z=800 \mathrm{~m}$. Being the RSU placed at the center of the ROI, if the VANET is completely connected, then the transmitted packet reaches all vehicles in at most 5 hops in all scenarios. This is confirmed by the results shown in Figure 12, discussed in the following. After the 5-th hop, the presence of vertical crossing roads has a relevant impact.

- In the absence of vertical crossing roads $\left(\mathrm{TL}_{1}^{\mathrm{h}}, \mathrm{TL}_{2}^{\mathrm{h}}\right.$, $\left.\mathrm{R}_{2}^{\mathrm{h}}, \mathrm{R}_{2}^{\mathrm{h}}\right)$, the delay increases steeply. This is due to the fact that most of the vehicles in the ROI have already received the packet and, therefore, drop newly received versions of the same packet. If a vehicle has not received the packet yet, it will have to wait longer.

- In the presence of vertical crossing roads $\left(\mathrm{TL}_{1}^{\mathrm{hv}}\right.$, $\left.\mathrm{TL}_{2}^{\mathrm{hv}}, \mathrm{R}_{2}^{\mathrm{hv}}, \mathrm{R}_{2}^{\mathrm{hv}}\right)$, the delay increases smoothly. This is due to the fact that the vehicles entering the ROI from the vertical roads may retransmit the packet and, therefore, keep the delay short. Recall, however, that in the presence of vertical crossing roads the RE is lower than in the absence of them. In other words, fewer vehicles receive the packet but, when they do, the delay is more shorter.

As anticipated above, in Figure 12 the reachability is shown as a function of the hop number, considering scenarios (a) with TLs and (b) with Rs. In both cases, two representative values of $\rho_{\mathrm{s}} z$, namely 5 veh (low vehicle density) and 20 veh (high vehicle density), are considered. Characteristic trends can be observed in both the cases with TLs and Rs. From Figure 12 (b)) we observe that in the scenarios with $\mathrm{Rs}$ without vertical roads $\left(\mathrm{R}_{1}^{\mathrm{h}}\right.$ and $\left.\mathrm{R}_{2}^{\mathrm{h}}\right)$ the value of $\rho_{\mathrm{s}} z$ has, basically, no impact. In all the other cases, namely, scenarios with TLs (Figure 12 (a)) and scenarios with Rs with vertical roads, it can be observed that, for each specific scenario, at the first hops, the RE is higher for $\rho_{\mathrm{s}} z=5 \mathrm{veh}$, whereas for larger number of hops it becomes higher for $\rho_{\mathrm{s}} z=20 \mathrm{veh}$. This behavior can me motivated as follows. With $\rho_{\mathrm{s}} z=5$ veh the network is weakly connected and a very small number of vehicles can be physically reached by the RSU. For this reason, at the very first hops almost all the reachable nodes receive the packet. On the contrary, due to the scarce connectivity in this condition it is very difficult to reach all the reachable nodes. We finally observe that, coherently with the results of Figure 11, in all cases, either with TLs or Rs, the maximum reachability is approximately achieved at the 5-th hop.

\section{Per-Flow Performance of IF}

In this section we try to characterize the maximum amount of transferable data from the RSU to the vehicles passing through the ROI, by measuring the throughput, as defined in Subsection IV-B. Our goal is to derive an optimal strategy for content distribution applications, in order to maximize the amount of transferred data, by tuning systems parameters such as $\lambda$ and PS. We assume that the RSU transmits, every $100 \mathrm{~s}$, a new information flux constituted by $N_{\mathrm{p}}=1000$ equal size packets. We have considered two values of $\lambda$, respectively 10 and $100 \mathrm{pck} / \mathrm{s}$, and two values of PS, respectively, 100 and 1000 bytes, leading to 4 different configurations. Since $N_{\mathrm{p}}$ is fixed, the duration of the transmission is solely determined by $\lambda(10$ or $100 \mathrm{~s})$, while the PS determines the total amount of transmitted information (100 Kbytes or 1 Mbytes). We observe that the configuration $(\lambda=100 \mathrm{pck} / \mathrm{s}$, $\mathrm{PS}=1000$ bytes) represents a saturation conditions since the RSU emits packets with a data rate of $800 \mathrm{Kbit} / \mathrm{s}(80 \%$ of the theoretical data rate of $1 \mathrm{Mbps})$. Conversely, the configuration ( $\lambda=10 \mathrm{pck} / \mathrm{s}, \mathrm{PS}=100$ bytes $)$ leads to an highly unsaturated condition, since the data rate is equal $8 \mathrm{Kbit} / \mathrm{s}(0.8 \%$ of the theoretical data rate). The remaining configurations $(\lambda=10 \mathrm{pck} / \mathrm{s}, \mathrm{PS}=1000$ bytes $)$ and $(\lambda=$ $100 \mathrm{pck} / \mathrm{s}, \mathrm{PS}=100$ bytes) are both characterized by a data rate of $80 \mathrm{Kbit} / \mathrm{s}$ ( $8 \%$ of the theoretical data rate), and they allow to evaluate the impact of the transmission duration (respectively 100 and $10 \mathrm{~s}$ ) on the throughput.

In all scenarios taken in account we set $\rho_{\mathrm{s}} z=20 \mathrm{veh}$, thus yielding to a per-road density $\rho_{\mathrm{s}}$ equal to $0.02 \mathrm{veh} / \mathrm{m}$. 


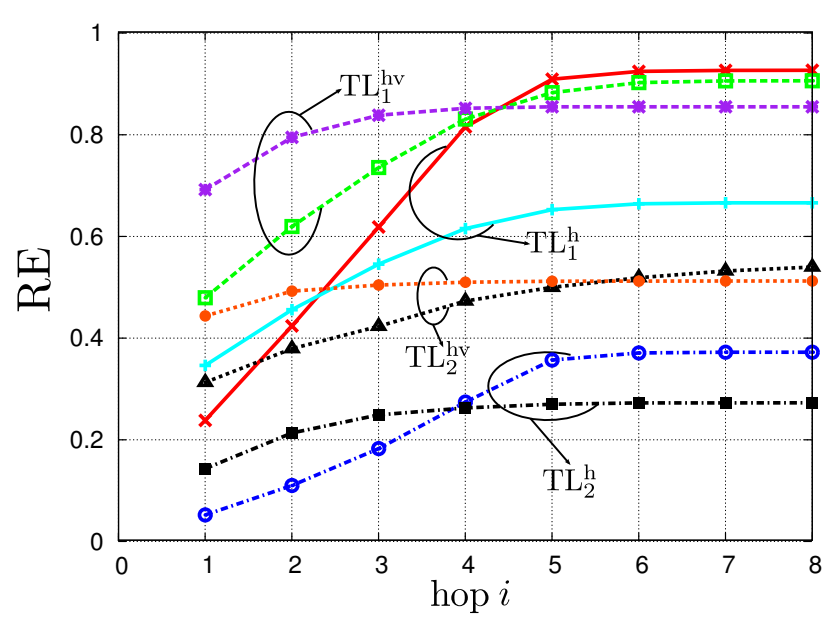

(a)

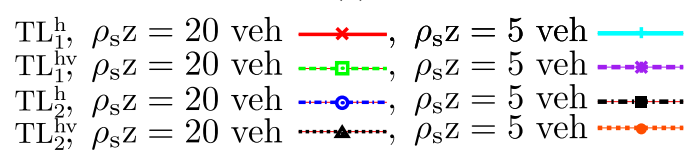

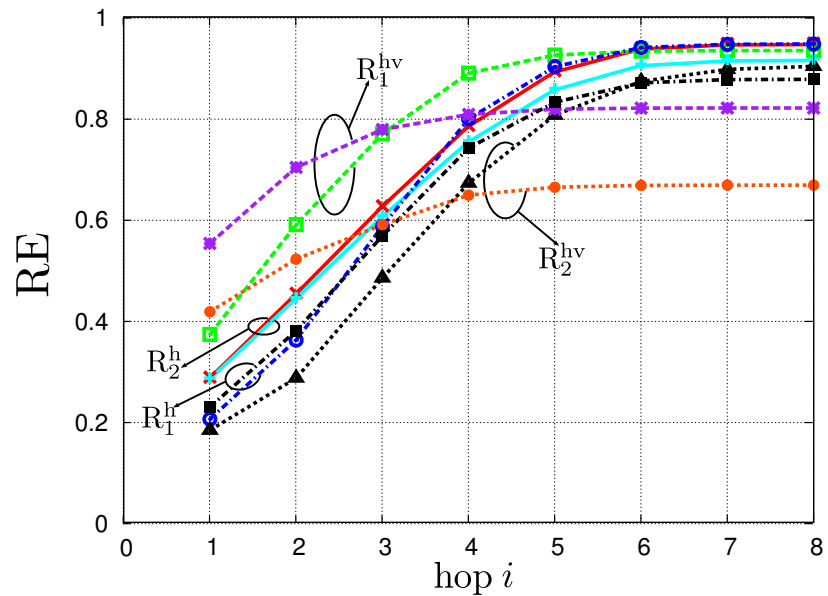

(b)

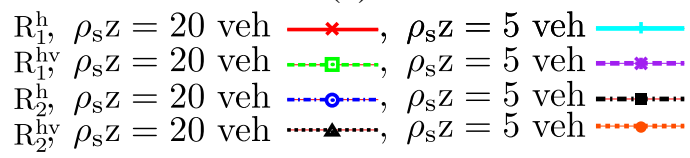

Figure 12. RE, as a function of the hop index, in scenarios (a) with TLs and (b) with Rs. In all cases, $\lambda=10$ pck/s, and $\rho_{\mathrm{s}} z=20$ veh.

Therefore, considering that $\ell_{\text {norm }}=10$, each scenario has a number of nodes give by $N=100\left(1+N_{\text {road }}^{\mathrm{v}}\right)$, but in $H_{\text {sta }}$ scenario, where $N=160$. The node indexes are assigned at the end of the simulation, after having ordered, decreasingly, the measured values. More precisely, vehicle 1 will have the highest throughput and the last vehicle will have the lowest one.

Finally, it has to be pointed out that in all the mobile scenarios there is a significant number of nodes with a zero throughput since they are lying, by construction, outside the ROI at the moment of the transmission. Moreover, the scenarios have typically a different number of vehicles $N$, because of the assumption of having the same average per-road vehicular density in all the horizontal and vertical roads. For these two reasons, in order to carry out a meaningful throughout comparison among scenarios with a different number of nodes, it is necessary to consider a normalized node index, defined as $i^{*}=\frac{i}{N}, i^{*} \in[0,1]$.

\section{A. Highway Scenarios}

In Figure 13, the throughput is shown, as a function of the normalized vehicle index $\left(i^{*}\right)$, considering (a) $\lambda=10 \mathrm{pck} / \mathrm{s}$ and (b) $\lambda=100 \mathrm{pck} / \mathrm{s}$. For each value of $\lambda$, we consider static $\left(\mathrm{H}_{\text {sta }}\right)$ and mobile $\left(\mathrm{H}_{\mathrm{mob}}\right)$ scenarios, and two values of PS (100 bytes and 1000 bytes). We observe that the saturated configuration (curves with PS $=1000$ bytes in Figure 13 (b)) has unsatisfactory performance on both mobile and static scenarios. On the opposite hand, the low-traffic load configuration $(\lambda=$ $10 \mathrm{pck} / \mathrm{s}, \mathrm{PS}=100$ bytes) shown in (Figure 13 (a), exhibits a throughput greater then 0.9 for all the vehicles in the ROI in both static and mobile scenarios. Therefore, we can consider the data rate of $8 \mathrm{Kbit} / \mathrm{s}(0.8 \%$ of the theoretical throughout) as the maximum sustainable rate.
From the results in Figure 13 (a) and Figure 13 (b), it emerges that the highest throughput (vehicle index 1) is obtained in the static $\left(\mathrm{H}_{\text {sta }}\right)$ scenarios. However, while in the mobile $\left(\mathrm{H}_{\mathrm{mob}}\right)$ scenarios the throughput experienced by all vehicles is similar, in the static scenarios there are relevant differences between the highest and lowest values. This is expected, as in a mobile scenario there are "less privileged" vehicles which stay longer near the RSU. However, this effect appears clearly only if the transmission duration is sufficiently long, as emerges by comparing the two configurations with a data rate equal to $80 \mathrm{Kbit} / \mathrm{s}$. In fact, when the transmission duration is only $10 \mathrm{~s}$ as in the case $(\lambda=100 \mathrm{pck} / \mathrm{s}$ and PS=100 bytes $)$ in Figure 13(b), the $H_{\text {mob }}$ scenario exhibits an unfair trend, similarly to the $\mathrm{H}_{\text {sta }}$. We finally observe that among the two $80 \mathrm{Kbits} / \mathrm{s}$ configurations does not emerge a clear winner, neither in the $\mathrm{H}_{\text {mob }}$ or $\mathrm{H}_{\text {sta }}$ scenario.

\section{B. Urban Scenarios}

In Figure 14 is shown the throughput as a function of the normalized node index $\left(i^{*}\right)$, obtained in urban scenarios with $N_{\text {jun }}=1$, considering both (a) TL and (b) $\mathrm{R}$ junctions. We have examined 4 combinations of PS and $\lambda$, by considering two values of PS (100 bytes and 1000 bytes) and two values of $\lambda(10 \mathrm{pck} / \mathrm{s}$ and $100 \mathrm{pck} / \mathrm{s})$. According to Figure 14, the throughput in the Rs scenario has very smooth variations and it behaves similar to the highway scenarios. This happens because a roundabout allow the vehicles to passing it without stopping, thus yielding to a more fluid vehicular traffic, reducing the local spatial-temporal variation of the vehicle density. In particular, in the scenario without vertical roads, the vehicles have always the priority at the junction and they never stop. Conversely, in the TLs scenario the spatial density significantly varies both in time and space, thus motivating the more irregular throughput shape exhibited 


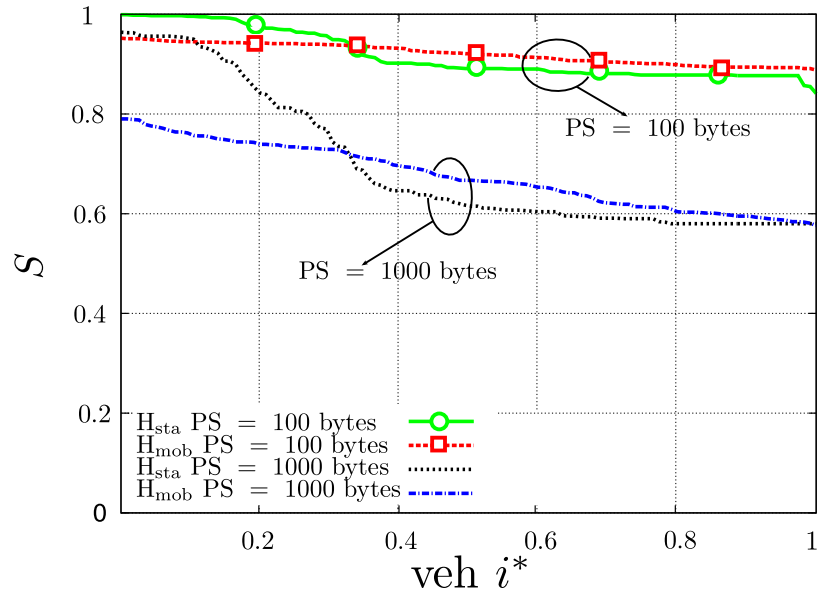

(a)

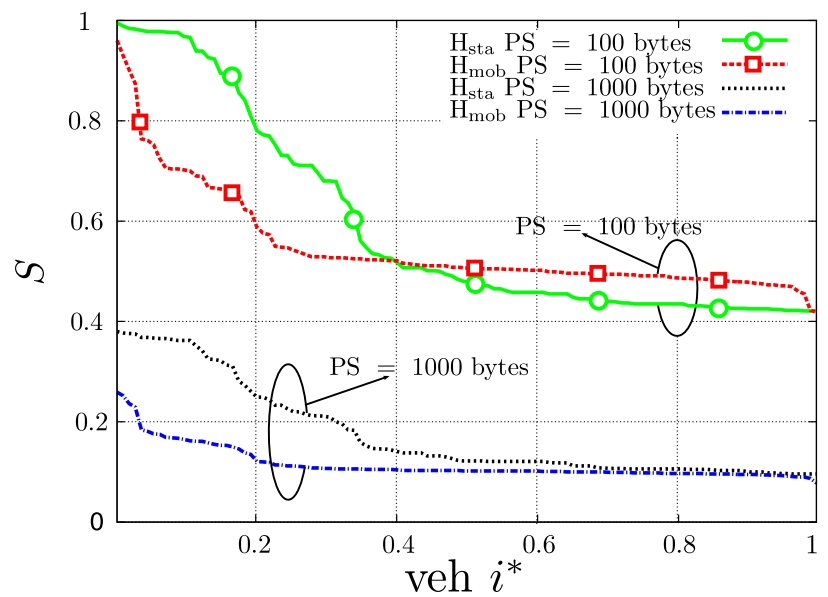

(b)

Figure 13. Throughput, as a function of the normalized vehicle index $\left(i^{*}\right)$, in $\mathrm{H}_{\text {sta }}$ and $\mathrm{H}_{\text {mob }}$ scenarios with two values of $\lambda$ : (a) 10 pck/s and (b) $100 \mathrm{pck} / \mathrm{s}$. In both cases, two values of PS are considered (100 bytes and 1000 bytes).

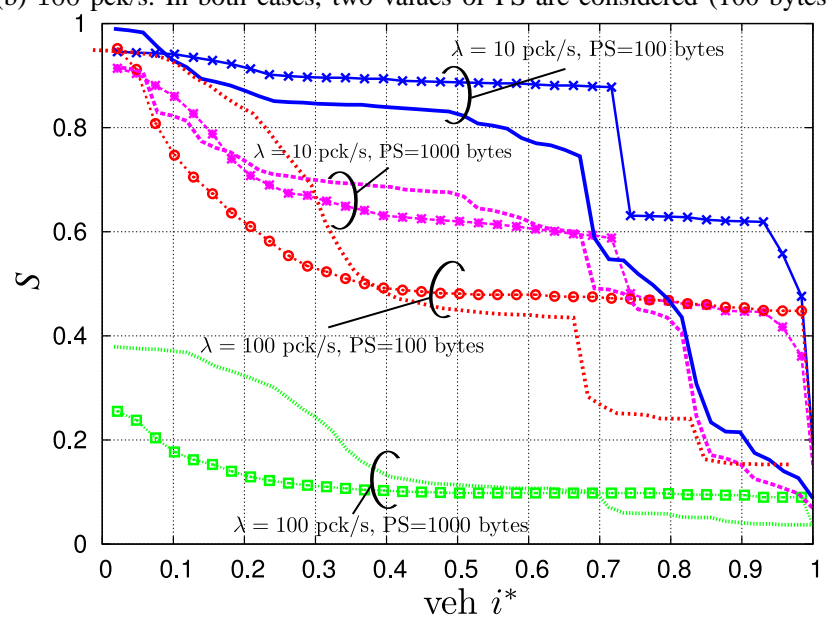

(a)

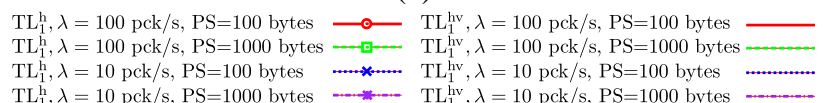

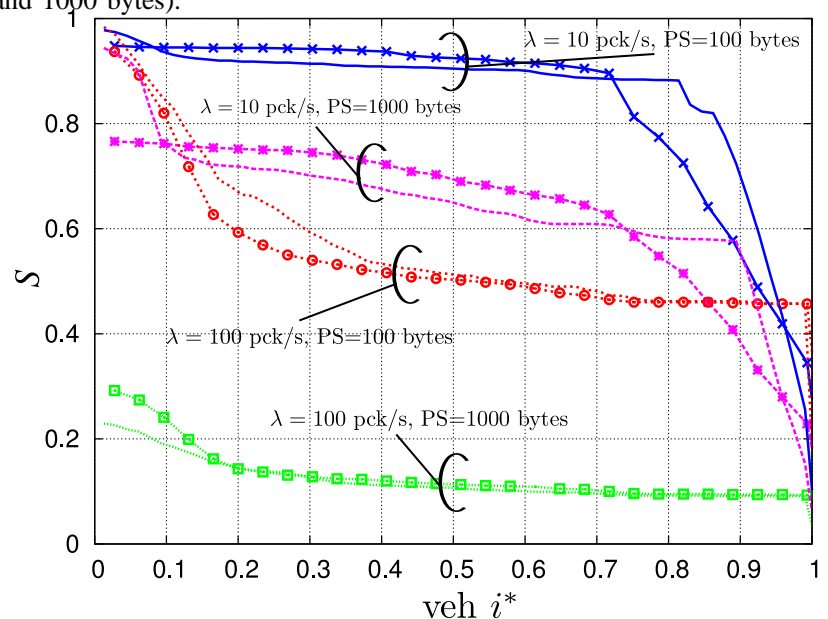

(b)

$\mathrm{R}_{1}^{\mathrm{h}}, \lambda=100 \mathrm{pck} / \mathrm{s}, \mathrm{PS}=100$ bytes $-\mathrm{C} \quad \mathrm{R}_{1}^{\mathrm{hv}}, \lambda=100 \mathrm{pck} / \mathrm{s}, \mathrm{PS}=100$ bytes

$\begin{array}{ll}\mathrm{R}_{1}^{\mathrm{h}}, \lambda=100 \mathrm{pck} / \mathrm{s}, \mathrm{PS}=100 \text { bytes }-1- & \mathrm{R}_{1}^{\mathrm{h}}, \lambda=100 \mathrm{pck} / \mathrm{s}, \mathrm{PS}=100 \text { bytes } \\ \mathrm{R}_{1}^{\mathrm{h}}, \lambda=100 \mathrm{pck} / \mathrm{s}, \mathrm{PS}=1000 \text { bytes ---回---. } & \mathrm{R}_{1}^{\text {hv }}, \lambda=100 \mathrm{pck} / \mathrm{s}, \mathrm{PS}=1000 \text { bytes }\end{array}$

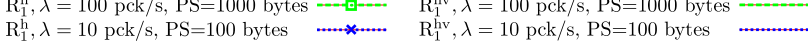

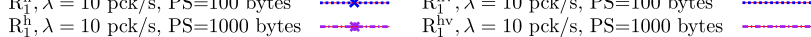

Figure 14. Throughput, as a function of the normalized vehicle index $\left(i^{*}\right)$ (a) in the $\mathrm{TL}_{1}^{\mathrm{h}}$ and $\mathrm{TL}_{1}^{\mathrm{hv}}$ scenarios and in (b) the $\mathrm{R}_{1}^{\mathrm{h}}$ and $\mathrm{R}_{1}^{\mathrm{hv}} \mathrm{scenarios}$. We have considered two values of PS, respectively 100 and 1000 bytes, and two values of $\lambda$, respectively,s 10 pck/s and 100 pck/s.

by the TLs scenarios. From Figure 14 we also observe that the Rs scenarios are insensitive with respect to the presence of a vertical roads, differently from the TLs scenarios which are significantly affected by the presence of a vertical road. We finally remark that among the configurations with the data rate of $80 \mathrm{Kbit} / \mathrm{s}$, in the Rs scenarios the configuration with $\lambda=10 \mathrm{pck} / \mathrm{s}$ exhibits a clear advantage with respect to the $\lambda=100 \mathrm{pck} / \mathrm{s}$ (as in the highway), while in the TLs scenarios, there is no a clear winners. Despite of these significant differences, the Rs and the Ts scenarios offer quite similar performance in terms of absolute value, especially in the scenarios without vertical roads.

\section{Comparative Analysis}

Finally, in Figure 15 we directly compare the throughput, as a function of the normalized node index, in highway and urban scenarios. In order to make a meaningful comparison, we only consider the urban scenarios without vertical crossing roads (i.e., $\mathrm{TL}_{1}^{\mathrm{h}}, \mathrm{TL}_{2}^{\mathrm{h}}, \mathrm{R}_{1}^{\mathrm{h}}$, and $\mathrm{R}_{2}^{\mathrm{h}}$ ). For easy of comprehension, we focus on a single configuration ( $\mathrm{PS}=1000$ bytes, $\lambda=10 \mathrm{pck} / \mathrm{s}$ ) characterized by transmitting a packet flow of 1 Mbytes in $100 \mathrm{~s}$, with a data rate of $80 \mathrm{Kbit} / \mathrm{s}$. Figure 15 offers many insights on the characteristics of the different analyzed scenarios. First of all, both Rs scenarios exhibit a trend similar to which of the $H_{\text {mob }}$ scenario, with slightly lower maximum values and slightly higher minimum values. This behavior is reasonable, since the Rs scenarios without vertical roads can be considered as a low-speed highway, with a flux of vehicles regular both in space and time.

Conversely, we observe that the $\mathrm{TL}_{1}^{\mathrm{h}}$ scenario behaves like the $\mathrm{H}_{\text {sta }}$ scenario with the exception of having slightly worse performance and a more irregular shape. This behavior can be easily motivated by considering that for approximately half of the transmission time the traffic lights are red, thus leading to the the formation of two clusters of static vehicles around the RSU.

Finally, we observe that $\mathrm{TL}_{2}^{\mathrm{h}}$ scenario has a different behavior from the other scenarios with smaller maximum 


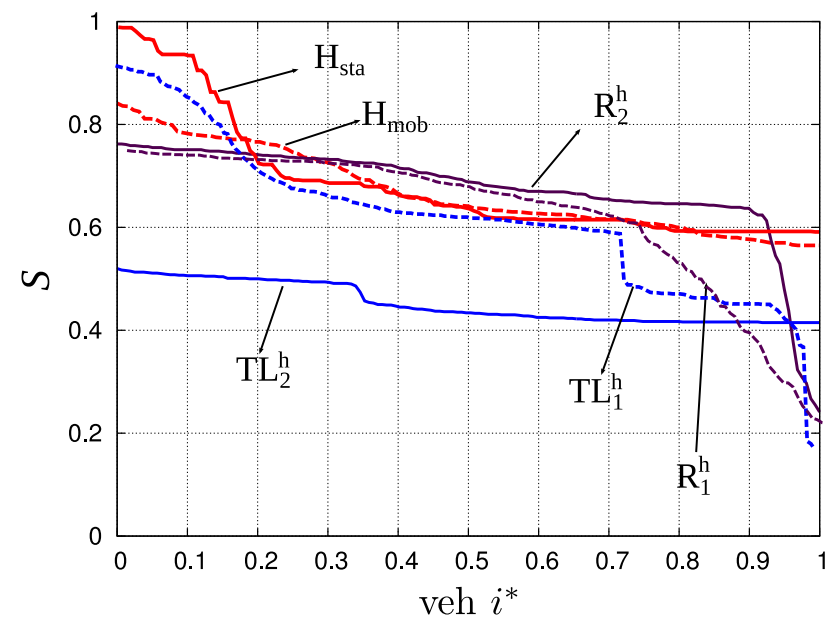

Figure 15. Throughput, as a function of the normalized vehicle index $\left(i^{*}\right)$ in various urban and highway scenarios. We consider $\mathrm{PS}=1000$ bytes and $\lambda=10 \mathrm{pck} / \mathrm{s}$.

and average throughput. This performance can be motivated by considering that the RSU is located in the midst between the two junctions, instead of being near the TLs as in the $\mathrm{TL}_{1}^{\mathrm{h}}$ scenario. In this condition, the RSU is in spatial region where the local spatial density is smaller than the average. For this reasons the retransmission process is weaker and it can be easily be interrupted because of the lack of connectivity.

\section{CONCLUSIONS}

In this paper, we have analyzed the impact of the vehicular mobility on the dissemination of broadcast data in $\mathrm{I} 2 \mathrm{~V}$ networks by means of numerical simulations, supported by realistic mobility models and by considering both highway and several types of urban scenarios. In all cases, communications have relied on the use of a probabilistic forwarding protocol, namely IF. The analysis has been carried out analyzing both per-packet and perflow system performance.

From a single packet perspective, the performance of a stateless broadcast protocol, such as IF, has shown to be insensitive to the vehicle mobility level, at least in highway scenarios. On the other hand, in urban scenarios the performance of the IF protocol is affected by the number and the type of functions (either TLs or Rs), since they induce significant spatial-temporal variations of the local vehicle density and of the VANET connectivity.

The information flow analysis has offered several insights on the impact of mobility on the performance of the IF protocol. Our simulations have shown that with an increasing vehicles mobility, both the throughput maximum and its variance reduces. We have also found some interesting similarities among the analyzed scenarios, namely, between urban roads with traffic lights and stationary highways, and between urban scenarios with roundabouts and mobile highways.

\section{REFERENCES}

[1] E. Schoch, F. Kargl, M. Weber, and T. Leinmuller, "Comm. patterns in VANETs," IEEE Commun. Mag., vol. 46, no. 11, pp. 2-8, Nov. 2008.
[2] Insitute of Electrical and Electronics Engineers, "IEEE1906.3-2007. Trial-Use Standard for Wireless Access in Vehicular Environments (WAVE) Networking Services," 2007.

[3] "ISO TC204 WG16," Website: http: //www.calm.hu/.

[4] M. Fiore and J. Härri, "The networking shape of vehicular mobility," in Proc. ACM Int. Symp. on Mobile Ad Hoc Network. and Comput. (MOBIHOC). New York, NY, USA: ACM, 2008, pp. 261-272.

[5] M. Brackstone and M. McDonald, "Car-following: a historical review," Transportation Research Part F: Traffic Psychology and Behaviour, vol. 2, no. 4, pp. 181 - 196, 1999.

[6] J. Härri, F. Filali, and C. Bonnet, "Performance comparison of AODV and OLSR in VANETs urban environments under realistic mobility patterns," in Proc. 5th IFIP Mediterranean Ad-Hoc Networking Workshop, Lipari, Italy, June 2006, pp. 301-309.

[7] S. Panichpapiboon and G. Ferrari, "Irresponsbile forwarding," in Proc. IEEE Intern. Conf. on Intelligent Transport System Telecommunication (ITST), Phuket, Thailand, Oct. 2008, pp. 311-316.

[8] S. Busanelli, G. Ferrari, and S. Panichpapiboon, "Efficient broadcasting in IEEE 802.11 networks through irresponsible forwarding," in Proc. IEEE Global Telecommun. Conf. (GLOBECOM), Honolulu, HA, USA, Dec. 2009.

[9] S. Busanelli, G. Ferrari, and V. A. Giorgio, "On the effects of mobility for efficient broadcast data dissemination in I2V networks," in Proc. SWiM Workshop IEEE Global Telecommun. Conf. (GLOBECOM), Miami, FL, USA, Dec. 2010.

[10] N. Wisitpongphan, F. Bai, P. Mudalige, V. Sadekar, and O. K. Tonguz, "Routing in sparse vehicular ad hoc wireless networks," IEEE J. Select. Areas Commun., vol. 25, no. 8, pp. 1538-1556, Oct. 2007.

[11] "VanetMobiSim Project," Website: http: / /vanet. eurecom. fr/.

[12] "SUMO Project,"

[13] M. Fiore, J. Härri, F. Filali, and C. Bonnet, "Vehicula mobility simulation for VANETs," in Proc. 40th Annual Simulation Symposium (ANSS'07), Norfolk, VA, USA, Mar. 2007, pp. 301-309.

[14] M. Treiber, A. Hennecke, and D. Helbing, "Congested traffic states in empirical observations and microscopic simulations," Phys. Rev. E, vol. 62, no. 2, pp. 1805-1824, Aug. 2000.

[15] F. Karnadi, Z. H. Mo, and K. chan Lan, "Rapid generation of realistic mobility models for VANET," in Proc. IEEE Wireless Communications and Networking Conference. (WCNC'07), Hong Kong, China, Mar. 2007, pp. 25062511.

[16] S. Krauss, P. Wagner, and C. Gawron, "Metastable states in a microscopic model of traffic flow," Physical Review $E$, vol. 55, no. 5, pp. 5597-5602, 1997.

[17] S. Ni, Y. Tseng, Y. Chen, and J. Sheu, "The broadcast storm problem in a mobile ad hoc network," in Proc. ACM Intern. Conf. on Mobile Comput. and Network. (MOBICOM), Seattle, USA, Aug. 1999, pp. 151-162.

[18] Insitute of Electrical and Electronics Engineers, "IEEE Std 802.11TM-2007. Part 11: Wireless LAN Medium Access Control (MAC) and Physical Layer (PHY) specifications," 2007.

[19] "Network Simulator 2 (ns-2)," Available at: http://isi.edu/nsnam/ns/. 